\title{
Efficient Numerical Algorithm for the Solution of Nonlinear Two- Dimensional Volterra Integral Equation Arising from Torsion Problem
}

\author{
A. M. Al-Bugami \\ Department of Mathematics, Faculty of Sciences, Taif University, Taif, Saudi Arabia \\ Correspondence should be addressed to A. M. Al-Bugami; abeer101aa@yahoo.com
}

Received 5 April 2021; Accepted 21 June 2021; Published 16 July 2021

Academic Editor: Giuseppe Pellicane

Copyright (C) 2021 A. M. Al-Bugami. This is an open access article distributed under the Creative Commons Attribution License, which permits unrestricted use, distribution, and reproduction in any medium, provided the original work is properly cited.

\begin{abstract}
In this article, an effective method is given to solve nonlinear two-dimensional Volterra integral equations of the second kind, which is arising from torsion problem for a long bar that consists of the nonlinear viscoelastic material type with a fixed elliptical cross section. First, the existence of a unique solution of this problem is discussed, and then, we find the solution of a nonlinear twodimensional Volterra integral equation (NT-DVIE) using block-by-block method (B-by-BM) and degenerate kernel method (DKM). Numerical examples are presented, and their results are compared with the analytical solution to demonstrate the validity and applicability of the method.
\end{abstract}

\section{Introduction}

The equations of the torsion problem were derived in detail with analytical solutions, by Muskhelishvili [1], Frank and Mises [2], Nowinski [3], and Sneddon and Berry [4]. The problem can be formulated as a boundary value problem of the Laplace equation. In [5], boundary element method was developed for the nonuniform torsion of simply or multiply connected cylindrical bars of arbitrary cross section, where the bar is subjected to an arbitrary distributed twisting moment while its edges are restrained by the most general linear torsional. In [6], nonlinear inelastic uniform torsion of bars by BEM was studied. Sapountzakis and Tsipiras in [7] used the boundary element method solution to the nonlinear inelastic uniform torsion problem of composite bars. El-Kalla and AL-Bugami in [8] discussed the nonlinear VolterraFredholm integral equation and torsion problems. Sheshtawy and Ghaleb in [9], discussed approximate solution to the problem of torsion by a boundary integral method. Assari, in [10], discussed the numerical solution of TDFIE of the second kind on nonrectangular domains. Fattahzadeh, in [11], solved two-dimensional linear and nonlinear Fredholm integral equations of the first kind based on Haar wavelet. Authors, in [12], solved twodimensional integral equation of the first kind by a multistep method. Alturk, in [13], solved two-dimensional Fredholm integral equations of the first kind using regularization-homotopy method. In this work, effective numerical methods are proposed to obtain the solution of nonlinear two-dimensional Volterra integral equations of the second kind and study the values of absolute errors.

\section{Basic Formulas}

While one end of the bar, of length $b$, is prevented from rotating, the other end is rotated about the $z$-axis. So that a section at distance $z$ from the fixed end turns through angle $\theta$, the variation of angle $\theta$ with $z, z \in[0, b]$, is taken as

$$
\theta=z \alpha(x, t) .
$$

$\alpha$ is a twist angle. The displacement $u_{\theta}$ of a particle in a tangential direction is given by

$$
u_{\theta}=r \theta \text {, }
$$


where $r$ is the radius of the particle. Then, we get

$$
\begin{aligned}
& u_{x}=-\left(\frac{y}{r}\right) u_{\theta}, \\
& u_{y}=\left(\frac{x}{r}\right) u_{\theta} .
\end{aligned}
$$

From (1), (2), and (3), we obtained

$$
\begin{gathered}
u_{x}=-y z \alpha(x, t), \\
u_{y}=x z \alpha(x, t), \\
u_{z}=\alpha(x, t) \psi(x, y) .
\end{gathered}
$$

Hence, we get

$$
\begin{gathered}
e_{x}=\frac{\partial u_{x}}{\partial x}=0, \\
e_{y}=\frac{\partial u_{y}}{\partial y}=0, \\
e_{z}=\frac{\partial u_{z}}{\partial z}=0, \\
e_{x y}=\frac{1}{2}\left(\frac{\partial u_{x}}{\partial y}+\frac{\partial u_{y}}{\partial x}\right)=0 \\
e_{z x}=\frac{1}{2}\left(\frac{\partial \psi}{\partial x}-y\right) \alpha \\
e_{z y}=\frac{1}{2}\left(\frac{\partial \psi}{\partial y}+x\right) \alpha, \\
\sigma_{z x}=\left(\frac{\partial \psi}{\partial x}-y\right) G \alpha \\
\sigma_{z y}=\left(\frac{\partial \psi}{\partial y}+x\right) G \alpha .
\end{gathered}
$$

The stress equilibrium equations are now examined:

$$
\begin{aligned}
& \frac{\partial \sigma_{x}}{\partial x}+\frac{\partial \sigma_{x y}}{\partial y}+\frac{\partial \sigma_{x z}}{\partial z}=0 \\
& \frac{\partial \sigma_{y}}{\partial y}+\frac{\partial \sigma_{y z}}{\partial z}+\frac{\partial \sigma_{y x}}{\partial x}=0 \\
& \frac{\partial \sigma_{z}}{\partial z}+\frac{\partial \sigma_{z x}}{\partial x}+\frac{\partial \sigma_{z y}}{\partial y}=0
\end{aligned}
$$

A comparison of (6) and (8) now gives

$$
\begin{gathered}
\frac{\partial \phi}{\partial y}=\frac{\partial \psi}{\partial x}-y \\
-\frac{\partial \phi}{\partial x}=\frac{\partial \psi}{\partial y}+x
\end{gathered}
$$

The functions $\theta$ and $\psi$ must satisfy the relations

$$
\begin{gathered}
\nabla^{2} \phi=-2, \\
\nabla^{2} \psi=0 .
\end{gathered}
$$

Stresses are derived from scalar $\phi$ in (6) in such a way that rectangular axes with any orientation may be used.

Let the origin of coordinate axes $(n, s)$ be situated on the boundary of the section, direction $n$ being normal to the boundary and directions being tangential to it. Local values of stresses are now given by

$$
\begin{aligned}
\sigma_{z n} & =\frac{\partial \phi}{\partial s} G \alpha, \\
\sigma_{z n} & =\frac{\partial \phi}{\partial n} G \alpha .
\end{aligned}
$$
by

The force $p_{x}$ acting on a vertical strip of width $\delta x$ is given

$$
p_{x}=G \alpha \delta x \int_{A}^{B} \frac{\partial \phi}{\partial y} d y
$$

It can be seen that the torque on the section is given by

$$
T=\iint_{R}\left(x \sigma_{z y}-y \sigma_{z x}\right) d x d y
$$

where $T$ is the moment of torque.

\section{Solution in the Form of NTVIE}

The deviator strain of nonlinear elastic material is as follows:

$$
\begin{aligned}
& E_{y z}=e_{y z}, \\
& E_{x z}=e_{x z} .
\end{aligned}
$$

Using (5) in (14), we get

$$
\begin{aligned}
& E_{y z}=\frac{1}{2}\left(\frac{\partial \psi}{\partial y}+x\right) \alpha(x, t), \\
& E_{x z}=\frac{1}{2}\left(\frac{\partial \psi}{\partial x}-y\right) \alpha(x, t) .
\end{aligned}
$$


The strain deviator tensor is defined as

$$
\begin{array}{cc}
E_{i j}=e_{i j^{-}} \frac{1}{3} e_{k k} \delta_{i j}, & e_{k k}=e_{x x}+e_{y y}+e_{z z}, i, j=x, y, z, \\
S_{i j}=\sigma_{i j^{-}} \frac{1}{3} \sigma_{k k} \delta_{i j}, & \sigma_{k k}=\sigma_{x x}+\sigma_{y y}+\sigma_{z z}, i, j=x, y, z .
\end{array}
$$

The second invariant of strain and stress tensor is as follows:

$$
E^{2}=e_{i j} e_{i j} \frac{1}{3} e_{k k} e_{k k}
$$

Using (5) in (17), we get

$$
E^{2}=2\left[e_{y z}^{2}+e_{z x}^{2}\right]
$$

Using (17) again, we get

$$
E^{2}=\frac{1}{2}\left[\left(\frac{\partial \psi(x, y)}{\partial y}+x\right)^{2}+\left(\frac{\partial \psi(x, y)}{\partial x}-y\right)^{2}\right] \alpha^{2}(x, t)
$$

Therefore, for the stress components, we find that $\sigma_{x z}, \sigma_{y z}$ are the only nonvanishing components of stress; thus, we find

$$
\sigma_{x x}=\sigma_{y y}=\sigma_{z z}=\sigma_{x y}=0 .
$$

Also,

$$
\begin{gathered}
S_{i i}=0, \\
S_{x z}=\sigma_{x z}, \\
S_{y z}=\sigma_{y z} .
\end{gathered}
$$

In addition, the principal cubic theory is given by

$S_{i j}=2 G E_{i j}+\int_{0}^{t} \int_{0}^{x} J(t-\tau, x-y) E_{i j} d y d \tau+\int_{0}^{t} \int_{0}^{x} k(t-\tau, x-y) E^{2} E_{i j} d y d \tau$,

where $G$ is the shear modulus of the material and $J(t-\tau, x$ $-y), k(t-\tau, x-y)$ are the kernel functions.

From (21) and (22),

$\sigma_{x z}=S_{x z}=2 G E_{x z}+\int_{0}^{t} \int_{0}^{x} J(t-\tau, x-y) E_{x z} d y d \tau+\int_{0}^{t} \int_{0}^{x} k(t-\tau, x-y) E^{2} E_{x z} d y d \tau$,

$\sigma_{y z}=S_{y z}=2 G E_{y z}+\int_{0}^{t} \int_{0}^{x} J(t-\tau, x-y) E_{y z} d y d \tau+\int_{0}^{t} \int_{0}^{x} k(t-\tau, x-y) E^{2} E_{y z} d y d \tau$.
Using (15) and (19) in (22), (23), and (24), we obtain

$$
\begin{aligned}
\sigma_{x z}= & G\left(\frac{\partial \psi}{\partial x}-y\right) \alpha(x, t)+\frac{1}{2}\left(\frac{\partial \psi}{\partial x}-y\right) \int_{0}^{t} \int_{0}^{x} J(t-\tau, x-y) \alpha(y, \tau) d y d \tau \\
& +\frac{1}{4}\left[\left(\frac{\partial \psi}{\partial x}-y\right)^{3}+\left(\frac{\partial \psi}{\partial y}+x\right)^{2}\left(\frac{\partial \psi}{\partial x}-y\right)\right] \\
& \cdot \int_{0}^{t} \int_{0}^{x} k(t-\tau, x-y) \alpha^{3}(y, \tau) d y d \tau .
\end{aligned}
$$

Also,

$$
\begin{aligned}
\sigma_{y z}= & \left(\frac{\partial \psi}{\partial y}+x\right)\left[G \alpha(x, t)+\frac{1}{2} \int_{0}^{t} \int_{0}^{x} J(t-\tau, x-y) \alpha(y, \tau) d y d \tau\right] \\
& +\frac{1}{4}\left[\left(\frac{\partial \psi}{\partial x}-y\right)^{2}+\left(\frac{\partial \psi}{\partial y}+x\right)^{2}\right] \int_{0}^{t} \int_{0}^{x} k(t-\tau, x-y) \alpha^{3}(y, \tau) d y d \tau
\end{aligned}
$$

Using (25) and (26) in (34), we have

$$
\begin{aligned}
T= & \left(\iint_{R}\left[x \frac{\partial \psi}{\partial y}-y \frac{\partial \psi}{\partial x}+x^{2}+y^{2}\right] d x d y\right) \\
& \cdot\left[G \alpha(x, t)+\frac{1}{2} \int_{0}^{t} \int_{0}^{x} J(t-\tau, x-y) \alpha d y d \tau\right]+\frac{1}{4} \\
& \cdot\left(\iint_{R}\left[x \frac{\partial \psi}{\partial y}-y \frac{\partial \psi}{\partial x}+x^{2}+y^{2}\right]\left[\left(\frac{\partial \psi}{\partial y}+x\right)^{2}+\left(\frac{\partial \psi}{\partial x}-y\right)^{2}\right] d x d y\right) \\
& \cdot\left(\int_{0}^{t} \int_{0}^{x} k(t-\tau, x-y) \alpha^{3}(y, \tau) d y d \tau\right) .
\end{aligned}
$$

Let

$$
\begin{gathered}
A_{1}=\iint_{R}\left[x \frac{\partial \psi}{\partial y}-y \frac{\partial \psi}{\partial x}+x^{2}+y^{2}\right] d x d y \\
\left.A_{1}=\iint_{R}\left[x \frac{\partial \psi}{\partial y}-y \frac{\partial \psi}{\partial x}+x^{2}+y^{2}\right]\left[\frac{\partial \psi}{\partial y}+x\right)^{2}+\left(\frac{\partial \psi}{\partial x}-y\right)^{2}\right] d x d y .
\end{gathered}
$$

Then, equation (27) becomes

$$
\begin{aligned}
T= & A_{1}\left(G \alpha(x, t)+\frac{1}{2} \int_{0}^{t} \int_{0}^{x} J(t-\tau, x-y) \alpha(y, \tau) d y d \tau\right. \\
& +\frac{1}{4} A_{2} \int_{0}^{t} \int_{0}^{x} k(t-\tau, x-y) \alpha^{3} d y d \tau .
\end{aligned}
$$

Then, we get

$$
\psi(x, y)=x y\left(\frac{b^{2}-a^{2}}{a^{2}+b^{2}}\right)
$$

where $a$ and $b$ are the semimajor axis of the ellipse. 
By calculating $\partial \psi / \partial x$ and $\partial \psi / \partial y$ from equation (31) and introducing the result in (29), we find

$$
A_{1}=\frac{1}{a^{2}+b^{2}} \iint_{R}\left(a^{2} y^{2}+b^{2} x^{2}\right) d x d y=\frac{\pi a^{3} b^{3}}{a^{2}+b^{2}}
$$

Also,

$$
A_{2}=\frac{2}{a^{2}+b^{2}} \iint_{R}\left(b^{2} x^{2}+a^{2} y^{2}\right)\left[\frac{4 b^{4} x^{2}}{\left(a^{2}+b^{2}\right)^{2}}+\frac{4 a^{4} y^{2}}{\left(a^{2}+b^{2}\right)^{2}}\right] d x d y
$$

Then, we have

$$
A_{2}=\frac{4 \pi a^{5} b^{5}}{3\left(a^{2}+b^{2}\right)^{2}}
$$

Here, $A_{1}$ is the torsional rigidity and $A_{2}$ is the polar moment of inertia of the cross section of the bar.

Write formula (27) in the form

$$
\begin{gathered}
G \alpha(x, t)+\frac{1}{2} \int_{0}^{t} \int_{0}^{x} J(t-\tau, x-y) \alpha(y, \tau) d y d \tau+\frac{A_{2}}{4 A_{1}} \int_{0}^{t} \int_{0}^{x} k(t-\tau, x-y) \alpha^{3} d y d \tau=\frac{T}{A_{1}}, \\
\lambda=\frac{2 G v}{(1-2 v)}, \\
K=\frac{2 G(1+v)}{3(1-2 v)} .
\end{gathered}
$$

Here, $v$ is the Poison ratio, and then, we obtain

$$
\begin{array}{r}
\alpha(x, t)+\lambda_{1} \int_{0}^{t} \int_{0}^{x} J(t-\tau, x-y) \alpha(y, \tau) d y d \tau \\
+\lambda_{2} \int_{0}^{t} \int_{0}^{x} k(t-\tau, x-y) \alpha^{3} d y d \tau=f(x, t),
\end{array}
$$

where $\lambda_{1}(\lambda / 2 G), \lambda_{2}\left(A_{2} K / 4 A_{1} G\right)$, and $f(x, t)=\left(T / A_{1} G\right)$.

Formula (36) represents NTVIE of the second kind, $J(\mid t$ $-\tau|| x-y \mid$,$) is the kernel of linear term, and k(|t-\tau|, \mid x-y$ |) is the kernel nonlinear term.

If the bar is a linear and viscoelastic materialk $(|t-\tau|,|x-y|)$, then we get

$$
\alpha(x, t)+\lambda_{1} \int_{0}^{t} \int_{0}^{x} J(t-\tau, x-y) \alpha(y, \tau) d y d \tau=f(x, t) .
$$

If the bar is a nonlinear and viscoelastic material, we get $J(|t-\tau|,|x-y|)=0$; then,

$$
\alpha(x, t)+\lambda_{2} \int_{0}^{t} \int_{0}^{x} k(t-\tau, x-y) \alpha^{3} d y d \tau=f(x, t) .
$$

The general form of formula (36) is

$$
\begin{aligned}
& \mu \alpha(x, t)+\lambda_{1} \int_{0}^{t} \int_{0}^{x} J(t-\tau, x-y) \alpha(y, \tau) d y d \tau \\
& \quad+\lambda_{2} \int_{0}^{t} \int_{0}^{x} k(t-\tau, x-y) \gamma(\tau, y, \alpha(y, \tau)) d y d \tau=f(x, t),
\end{aligned}
$$

where-

$\lambda_{1}=\lambda / 2 \mu, \lambda_{2}=A_{2} K / 4 A_{1} \mu$, and $f(x, t)=T / A_{1} \mu \cdot \gamma(y, \tau, \alpha(y, \tau$ )$), f(x, t) \in L_{2}[0, b] \times C[0, T]$, are given continuous functions and $\alpha(x, t)$ is an unknown function. $\lambda_{1}, \lambda_{2}$ known constants, which have many physical meaning, may be complex. $J(\mid t$ $-\tau|| x-y \mid$,$) and k(|t-\tau|,|x-y|)$ are continuous.

\section{The Existence of a Unique Solution of T-DVIE}

To discuss the existence and uniqueness solution of equation (39), we write it in the integral operator form

$$
\bar{Q} \alpha(x, t)=\frac{1}{\mu} f(x, t)+\frac{1}{\mu} Q \alpha(x, t) \quad(\mu \neq 0),
$$

where

$$
Q \alpha(x, t)=Q_{1} \alpha(x, t)+Q_{2} \alpha(x, t) \quad(\mu \neq 0),
$$

$$
\begin{gathered}
Q_{1} \alpha(x, t)=\lambda_{1} \int_{0}^{t} \int_{0}^{x} J(|t-\tau|,|x-y|) \alpha(y, \tau) d y d \tau, \\
Q_{2} \alpha(x, t)=\lambda_{2} \int_{0}^{t} \int_{0}^{x} k(|t-\tau|,|x-y|) \gamma(\tau, y, \alpha(y, \tau)) d y d \tau
\end{gathered}
$$

In addition, we assume the following conditions:

(1) $J(|t-\tau|,|x-y|)$ and $k(|t-\tau|,|x-y|) \in L_{2}[0, b] \times C[0$ , $T$ and satisfies $J(|t-\tau|,|x-y|) \leq M_{1}$ and $k(|t-\tau|,|x-y|) \leq M_{2}, M_{1}, M_{2}$ constants, $M>$ $M_{1}, M>M_{2} \forall t, \tau \in[0, T], x, y \in[0, b]$

(2) $f(x, t)$, with its partial derivatives with respect to $x$ and $t$, is continuous in $L_{2}[0, b] \times C[0, T]$, and its norm is defined as

$$
\|f(x, t)\|=\max _{0 \leq t \leq T}\left|\int_{0}^{t}\left\{\int_{0}^{x} f^{2}(x, \tau) d x\right\}^{1 / 2} d \tau\right|=G \quad(G \text { is a constant })
$$

(3) The known continuous function $\gamma(\mathrm{y}, \tau, \alpha(y, \tau))$ satisfies, for the constants $A>A_{1}, A>P$, the following 
conditions:

$$
\begin{gathered}
a-\|\gamma(\mathrm{x}, \mathrm{t}, \alpha(x, t))\| \leq A_{1}\|\alpha(x, t)\|, \\
b-\left\|\gamma(\mathrm{x}, \mathrm{t}, \alpha(x, t))-\gamma\left(\mathrm{x}, \mathrm{t}, \alpha_{2}(x, t)\right)\right\| \leq N(t, x)\left\|\alpha_{1}(x, t)-\alpha_{2}(x, t)\right\|, \\
\|N(t, x)\|_{L_{2}[0, b] \times C[0, T]}=\max _{0 \leq t \leq T}\left|\int_{0}^{t}\left\{\int_{0}^{x} N^{2}(\tau, x) d x\right\}^{1 / 2} d \tau\right|=P<\infty
\end{gathered}
$$

(4) The unknown function $\alpha(x, t)$ satisfies the Lipchitz condition for the first argument of position and Hö lder condition for the second argument of time, where

$$
\|\alpha(x, t)\|=\max _{0 \leq t \leq T}\left|\int_{0}^{t}\left\{\int_{0}^{x}|\alpha(x, t)|^{1 / 2} d x\right\}^{1 / 2} d \tau\right|
$$

(5) The kernels satisfies the Lipchitz condition with respect to position and Hölder condition with respect to time, where

$$
\begin{aligned}
& \left|J\left(|t-\tau|,\left|x_{1}-y_{1}\right|\right)-J\left(|t-\tau|,\left|x_{2}-y_{2}\right|\right)\right| \leq L_{1}|| x_{1}-y_{1}|-| x_{2}-y_{2}||, \\
& \left|k\left(|t-\tau|,\left|x_{1}-y_{1}\right|\right)-k\left(|t-\tau|,\left|x_{2}-y_{2}\right|\right)\right| \leq L_{1}|| x_{1}-y_{1}|-| x_{2}-y_{2}||, \\
& \left|J\left(\left|t_{1}-\tau_{1}\right|,|x-y|\right)-J\left(\left|t_{2}-\tau_{2}\right|,|x-y|\right)\right| \leq L_{2}|| t_{1}-\tau_{1}|-| t_{2}-\tau_{2}||, \\
& \left|k\left(\left|t_{1}-\tau_{1}\right|,|x-y|\right)-k\left(\left|t_{2}-\tau_{2}\right|,|x-y|\right)\right| \leq L_{2}|| t_{1}-\tau_{1}|-| t_{2}-\tau_{2}|| .
\end{aligned}
$$

Theorem 1. If conditions (1)-(3) are satisfied, then an equation has a unique solution in the Banach space $L_{2}[0, b] \times C[0$ $, T]$.

Lemma 2. Under the conditions (1)-((3)-a), the operator $\bar{Q}$ maps the space $L_{2}[0, b] \times C[0, T]$ into itself.

Proof. In the light of the two formulas (40) and (42), we have

$$
\begin{aligned}
\|\bar{Q} \alpha(x, t)\| \leq & \frac{1}{\mu}\|f(x, t)\|+\frac{\left|\lambda_{1}\right|}{|\mu|}\left\|\int_{0}^{t} \int_{0}^{x}|J(|t-\tau|,|x-y|)||\alpha(y, \tau)| d y d \tau\right\| \\
& +\frac{\left|\lambda_{2}\right|}{|\mu|}\left\|\int_{0}^{t} \int_{0}^{x}|k(|t-\tau|,|x-y|)|\right\| \gamma(\tau, y, \alpha(y, \tau))\|d y d \tau\| .
\end{aligned}
$$

Using conditions (1) and (2), then applying CauchySchwarz inequality, we get

$$
\begin{aligned}
\|\bar{Q} \alpha(x, t)\| \leq & \frac{G}{\mu}+\frac{\left|\lambda_{1}\right|}{|\mu|} M_{1}\left\|\int_{0}^{t} \int_{0}^{x}|\alpha(y, \tau)| d y d \tau\right\| \\
& +\frac{\left|\lambda_{2}\right|}{|\mu|} M_{2}\left\|\int_{0}^{t} \int_{0}^{x}\right\| \gamma(\tau, y, \alpha(y, \tau))\|d y d \tau\| .
\end{aligned}
$$

In view of condition $(3-a)$, the above inequality takes the form

$$
\begin{aligned}
& \|\bar{Q} \alpha(x, t)\| \leq \frac{G}{\mu}+\frac{\left|\lambda_{1}\right|}{|\mu|} M_{1} T\|\alpha(x, t)\|+\frac{\left|\lambda_{2}\right|}{|\mu|} M_{2} A T\|\alpha(x, t)\|, \\
& \|\bar{Q} \alpha(x, t)\| \leq \frac{G}{\mu}+\sigma\|\alpha(x, t)\|,\left(\sigma=\frac{\left|\lambda_{1}\right|}{|\mu|} M_{1} T+\frac{\left|\lambda_{2}\right|}{|\mu|} M_{2} A T\right), \quad T=\max _{0 \leq t \leq T} t .
\end{aligned}
$$

Inequality (49) shows that the operator $\bar{Q}$ maps the ball $S_{\rho}$ into itself, where

$$
\rho=\frac{G}{\left[|\mu|-\left|\lambda_{1}\right| M_{1} T+\left|\lambda_{2}\right| M_{2} A T\right]} .
$$

Since $\rho>0$ and $G>0$, therefore we have $\sigma<1$. Moreover, the inequality (49) involves the boundedness of the operator $Q$ of equation (42), where

$$
\|Q \alpha(x, t)\| \leq \sigma\|\alpha(x, t)\| .
$$

In addition, the inequalities (49) and (51), define the boundedness of the operator $Q$.

Lemma 3. Assume that the conditions (1) and (3-b) are verified, and then, $\bar{Q}$ is a contraction operator in the space $L_{2}[0$, $b] \times C[0, T]$.

Proof. For $\alpha_{1}(x, t)$ and $\alpha_{2}(x, t)$ in the space $L_{2}[0, b] \times C[0, T]$ and from equations (40) and (42), we find

$$
\begin{aligned}
\left\|\bar{Q} \alpha_{1}(x, t)-\bar{Q} \alpha_{2}(x, t)\right\| \leq & \frac{\left|\lambda_{1}\right|}{|\mu|}\left\|\int_{0}^{t} \int_{0}^{x}|J(|t-\tau|,|x-y|)|\left|\alpha_{1}(y, \tau)-\alpha_{2}(y, \tau)\right| d y d \tau\right\| \\
& +\frac{\left|\lambda_{2}\right|}{|\mu|}\left\|\int_{0}^{t} \int_{0}^{x} \mid k(|t-\tau|,|x-y|)\right\| \gamma\left(\tau, y, \alpha_{1}(y, \tau)\right) \\
& -\gamma\left(\tau, y, \alpha_{2}(y, \tau)\right) \mid d y d \tau \| .
\end{aligned}
$$

With the aid of conditions (1) and (3-b), the above inequality becomes

$$
\begin{aligned}
\left\|\bar{Q} \alpha_{1}(x, t)-\bar{Q} \alpha_{2}(x, t)\right\| \leq & \frac{\left|\lambda_{1}\right|}{|\mu|} M_{1}\left\|\int_{0}^{t} \int_{0}^{x}\left|\alpha_{1}(y, \tau)-\alpha_{2}(y, \tau)\right| d y d \tau\right\| \\
& +\frac{\left|\lambda_{2}\right|}{|\mu|} M_{2}\left\|\int_{0}^{t} \int_{0}^{x} N(\tau, y)\left|\alpha_{1}(y, \tau)-\alpha_{2}(y, \tau)\right| d y d \tau\right\| .
\end{aligned}
$$

Then, we get

$$
\left\|\bar{Q} \alpha_{1}(x, t)-\bar{Q} \alpha_{2}(x, t)\right\| \leq \sigma\left\|\alpha_{1}(x, t)-\alpha_{2}(x, t)\right\| .
$$

From inequality (54), we see that $\bar{Q}$ is continuous in the space $L_{2}[0, b] \times C[0, T]$, and then, $\bar{Q}$ is a contraction operator under the condition $\sigma<1$. 
TABLE 1: The approximate values and the absolute relative error values using B-by-BM for linear case $(k=1)$.

\begin{tabular}{|c|c|c|c|c|c|c|c|c|}
\hline \multirow{2}{*}{$N$} & \multirow{2}{*}{$t$} & \multirow{2}{*}{$x$} & \multicolumn{2}{|c|}{$v=0.21$} & \multicolumn{2}{|c|}{$v=0.27$} & \multicolumn{2}{|c|}{$v=0.33$} \\
\hline & & & Approx. $_{\text {BM }}$ & Error $_{\mathrm{BM}}$ & Approx. $_{\text {BM }}$ & Error $_{B M}$ & Approx. $_{\text {BM }}$ & Error $_{\mathrm{BM}}$ \\
\hline \multirow{9}{*}{10} & \multirow{3}{*}{0.2} & 0.2 & $1.31402 E-07$ & $2.90025 E-08$ & $1.49416 E-07$ & $4.70166 E-08$ & $1.80146 E-07$ & $7.77464 E-08$ \\
\hline & & 0.6 & $2.26330 E-04$ & $2.38155 E-06$ & $2.27809 E-04$ & $3.86090 E-06$ & $2.30333 E-04$ & $6.38472 E-06$ \\
\hline & & 1.0 & $8.02121 E-03$ & $2.12185 E-05$ & $8.03440 E-03$ & $3.44078 E-05$ & $8.05692 E-03$ & $5.69251 E-05$ \\
\hline & \multirow{3}{*}{0.6} & 0.2 & $5.13803 E-06$ & $2.37323 E-06$ & $6.61209 E-06$ & $3.84729 E-06$ & $9.12666 E-06$ & $6.36186 E-06$ \\
\hline & & 0.6 & $6.25662 E-03$ & $2.10002 E-04$ & $6.38706 E-03$ & $3.40451 E-04$ & $6.60961 E-03$ & $5.63002 E-04$ \\
\hline & & 1.0 & $2.17971 E-01$ & $1.97126 E-03$ & $2.19196 E-01$ & $3.19676 E-03$ & $2.21289 E-01$ & $5.28929 E-03$ \\
\hline & \multirow{3}{*}{1} & 0.2 & $3.14827 E-05$ & $1.86827 E-05$ & $4.30869 E-05$ & $3.02869 E-05$ & $6.28823 E-05$ & $5.00823 E-05$ \\
\hline & & 0.6 & $2.98837 E-02$ & $1.89016 E-03$ & $3.10579 E-02$ & $3.06430 E-03$ & $3.30610 E-02$ & $5.06746 E-03$ \\
\hline & & 1.0 & $1.0208 E+00$ & $2.08810 E-02$ & $1.0338 E+00$ & $3.38655 E-02$ & $1.0560 E+00$ & $5.60418 E-02$ \\
\hline \multirow{9}{*}{20} & \multirow{3}{*}{0.2} & 0.2 & $1.31402 E-07$ & $2.90026 E-08$ & $1.49416 E-07$ & $4.70166 E-08$ & $1.80146 E-07$ & $7.77464 E-08$ \\
\hline & & 0.6 & $2.26322 E-04$ & $2.37323 E-06$ & $2.27796 E-04$ & $3.84729 E-06$ & $2.30310 E-04$ & $6.36186 E-06$ \\
\hline & & 1.0 & $8.01883 E-03$ & $1.88375 E-05$ & $8.03053 E-03$ & $3.05385 E-05$ & $8.05049 E-03$ & $5.04999 E-05$ \\
\hline & \multirow{3}{*}{0.6} & 0.2 & $5.13803 E-06$ & $2.37323 E-06$ & $6.61209 E-06$ & $3.84729 E-06$ & $9.12666 E-06$ & $6.36186 E-06$ \\
\hline & & 0.6 & $6.25636 E-03$ & $2.09746 E-04$ & $6.38664 E-03$ & $3.40023 E-04$ & $6.60887 E-03$ & $5.62261 E-04$ \\
\hline & & 1.0 & $2.17893 E-01$ & $1.89313 E-03$ & $2.19069 E-01$ & $3.06904 E-03$ & $2.21075 E-01$ & $5.07511 E-03$ \\
\hline & \multirow{3}{*}{1} & 0.2 & $3.14827 E-05$ & $1.86827 E-05$ & $4.30869 E-05$ & $3.02869 E-05$ & $6.28823 E-05$ & $5.00823 E-05$ \\
\hline & & 0.6 & $2.98822 E-02$ & $1.88869 E-03$ & $3.10554 E-02$ & $3.06180 E-03$ & $3.30565 E-02$ & $5.06297 E-03$ \\
\hline & & 1.0 & $1.0203 E+00$ & $2.03892 E-02$ & $1.0330 E+00$ & $3.30540 E-02$ & $1.0546 E+00$ & $5.46597 E-02$ \\
\hline
\end{tabular}

TABLE 2: The approximate values and the absolute relative error values using B-by-BM for nonlinear case $(k=2)$.

\begin{tabular}{|c|c|c|c|c|c|c|c|c|}
\hline \multirow{2}{*}{$N$} & \multirow{2}{*}{$t$} & \multirow{2}{*}{$x$} & \multicolumn{2}{|c|}{$v=0.21$} & \multicolumn{2}{|c|}{$v=0.27$} & \multicolumn{2}{|c|}{$v=0.33$} \\
\hline & & & Approx. & Error $_{\mathrm{BM}}$ & Approx. ${ }_{\text {BM }}$ & Error $_{\text {BM }}$ & Approx. ${ }_{\text {BM }}$ & Error $_{\text {BM }}$ \\
\hline \multirow{9}{*}{10} & \multirow{3}{*}{0.2} & 0.2 & $1.02402 E-07$ & $2.89760 E-12$ & $1.02403 E-07$ & $3.83470 E-12$ & $1.02405 E-07$ & $5.43320 E-12$ \\
\hline & & 0.6 & $2.23988 E-04$ & $3.99838 E-08$ & $2.24001 E-04$ & $5.29166 E-08$ & $2.24023 E-04$ & 7.49807E-08 \\
\hline & & 1.0 & $8.01059 E-03$ & $1.05942 E-05$ & $8.01402 E-03$ & $1.40251 E-05$ & $8.01988 E-03$ & $1.98833 E-05$ \\
\hline & \multirow{3}{*}{0.6} & 0.2 & $2.77120 E-06$ & $6.40799 E-09$ & $2.77328 E-06$ & $8.48029 E-09$ & $2.77681 E-06$ & $1.20153 E-08$ \\
\hline & & 0.6 & $6.06303 E-03$ & $1.64133 E-05$ & $6.06834 E-03$ & $2.17224 E-05$ & $6.07739 E-03$ & $3.07804 E-05$ \\
\hline & & 1.0 & $2.16985 E-01$ & $9.85292 E-04$ & $2.17304 E-01$ & $1.30451 E-03$ & $2.17849 E-01$ & $1.84975 E-03$ \\
\hline & \multirow{3}{*}{1} & 0.2 & $1.30339 E-05$ & $2.33973 E-07$ & $1.31096 \mathrm{E}-05$ & $3.09638 E-07$ & $1.32387 E-05$ & $4.38713 E-07$ \\
\hline & & 0.6 & $2.86493 E-02$ & $6.55769 E-04$ & $2.88614 E-02$ & $8.67894 E-04$ & $2.92234 E-02$ & $1.22981 E-03$ \\
\hline & & 1.0 & $1.0352 E+00$ & $3.52274 E-02$ & $1.0466 E+00$ & $4.66473 E-02$ & $1.0661 E+00$ & $6.61596 E-02$ \\
\hline \multirow{9}{*}{20} & \multirow{3}{*}{0.2} & 0.2 & $1.02402 E-07$ & $2.92220 E-12$ & $1.02403 E-07$ & $3.86720 E-12$ & $1.02405 E-07$ & $5.47920 E-12$ \\
\hline & & 0.6 & $2.23955 E-04$ & $6.40800 E-09$ & $2.23957 E-04$ & $8.48030 E-09$ & $2.23960 E-04$ & $1.20154 E-08$ \\
\hline & & 1.0 & $8.00086 E-03$ & $8.66158 E-07$ & $8.00114 E-03$ & $1.14629 E-06$ & $8.00162 E-03$ & $1.62419 E-06$ \\
\hline & \multirow{3}{*}{0.6} & 0.2 & $2.77120 E-06$ & $6.40866 E-09$ & $2.77328 E-06$ & $8.48118 E-09$ & $2.77681 E-06$ & $1.20166 E-08$ \\
\hline & & 0.6 & $6.06202 E-03$ & $1.54093 E-05$ & $6.06701 E-03$ & $2.03926 E-05$ & $6.07551 E-03$ & $2.88934 E-05$ \\
\hline & & 1.0 & $2.16668 E-01$ & $6.68128 E-04$ & $2.16884 E-01$ & $8.84218 E-04$ & $2.17252 E-01$ & $1.25286 E-03$ \\
\hline & \multirow{3}{*}{1} & 0.2 & $1.30339 E-05$ & $2.33976 E-07$ & $1.74931 E-06$ & $3.09642 E-07$ & $1.32387 E-05$ & $4.38719 E-07$ \\
\hline & & 0.6 & $2.86437 E-02$ & $6.50129 E-05$ & $2.88539 E-02$ & $8.60376 E-04$ & $2.92126 E-02$ & $1.21903 E-03$ \\
\hline & & 1.0 & $1.0332 E+00$ & $3.32083 E-02$ & $1.0439 E+00$ & $4.39488 E-02$ & $1.0622 E+00$ & $6.22722 E-02$ \\
\hline
\end{tabular}


TABLE 3: The approximate values and the absolute relative error values using DKM for linear case $(k=1)$.

\begin{tabular}{|c|c|c|c|c|c|c|c|c|}
\hline \multirow{2}{*}{$N$} & \multirow{2}{*}{$t$} & \multirow{2}{*}{$x$} & \multicolumn{2}{|c|}{$v=0.21$} & \multicolumn{2}{|c|}{$v=0.27$} & \multicolumn{2}{|c|}{$v=0.33$} \\
\hline & & & Approx. $_{\text {DKM }}$ & Error $_{\mathrm{DKM}}$ & Approx.DKM & Error $_{\mathrm{DKM}}$ & Approx.DKM & Error $_{\mathrm{DKM}}$ \\
\hline \multirow{9}{*}{10} & \multirow{3}{*}{0.2} & 0.2 & $1.31400 E-07$ & $2.90000 E-08$ & $1.49413 E-07$ & $4.70130 E-08$ & $1.80140 E-07$ & $7.77400 E-08$ \\
\hline & & 0.6 & $2.26287 E-04$ & $2.33900 E-06$ & $2.27741 E-04$ & $3.79300 E-06$ & $2.30223 E-04$ & $6.27500 E-06$ \\
\hline & & 1.0 & $8.01120 E-03$ & $1.12000 E-05$ & $8.01863 E-03$ & $1.86300 E-05$ & $8.03201 E-03$ & $3.20100 E-05$ \\
\hline & \multirow{3}{*}{0.6} & 0.2 & $5.13839 E-06$ & $2.37359 E-06$ & $6.61170 E-06$ & $3.84690 E-06$ & $9.12583 E-06$ & $6.36103 E-06$ \\
\hline & & 0.6 & $6.25352 E-03$ & $2.06910 E-04$ & $6.38227 E-03$ & $3.35660 E-04$ & $6.60223 E-03$ & $5.55620 E-04$ \\
\hline & & 1.0 & $2.17224 E-01$ & $1.22400 E-03$ & $2.18091 E-01$ & $2.09100 E-03$ & $2.19685 E-01$ & $3.68500 E-03$ \\
\hline & \multirow{3}{*}{1} & 0.2 & $3.14808 E-05$ & $1.86808 E-05$ & $4.30829 E-05$ & $3.02829 E-05$ & $6.28735 E-05$ & $5.00735 E-05$ \\
\hline & & 0.6 & $2.98581 E-02$ & $1.86450 E-03$ & $3.10193 E-02$ & $3.02570 E-03$ & $3.30035 E-02$ & $5.00990 E-03$ \\
\hline & & 1.0 & $1.0141 E+00$ & $1.41000 E-02$ & $1.0244 E+00$ & $2.44000 E-02$ & $1.0431 E+00$ & $4.31000 E-02$ \\
\hline \multirow{9}{*}{20} & \multirow{3}{*}{0.2} & 0.2 & $1.31400 E-07$ & $2.90000 E-08$ & $1.49413 E-07$ & $4.70130 E-08$ & $1.80140 E-07$ & $7.77400 E-08$ \\
\hline & & 0.6 & $2.26287 E-04$ & $2.33900 E-06$ & $2.27741 E-04$ & $3.79300 E-06$ & $2.30223 E-04$ & $6.27500 E-06$ \\
\hline & & 1.0 & $8.01120 E-03$ & $1.12000 E-05$ & $8.01863 E-03$ & $1.86300 E-05$ & $8.03201 E-03$ & $3.20100 E-05$ \\
\hline & \multirow{3}{*}{0.6} & 0.2 & $5.13783 E-06$ & $2.37303 E-06$ & $6.61170 E-06$ & $3.84690 E-06$ & $9.12583 E-06$ & $6.36103 E-06$ \\
\hline & & 0.6 & $6.25352 E-03$ & $2.06910 E-04$ & $6.38227 E-03$ & $3.35660 E-04$ & $6.60223 E-03$ & $5.55620 E-04$ \\
\hline & & 1.0 & $2.17224 E-01$ & $1.22400 E-03$ & $2.18091 E-01$ & $2.09100 E-03$ & $2.19685 E-01$ & $3.68500 E-03$ \\
\hline & \multirow{3}{*}{1} & 0.2 & $3.14808 E-05$ & $1.86808 E-05$ & $4.30829 E-05$ & $3.02829 E-05$ & $6.28735 E-05$ & $5.00735 E-05$ \\
\hline & & 0.6 & $2.98581 E-02$ & $1.86450 E-03$ & $3.10193 E-02$ & $3.02570 E-03$ & $3.30035 E-02$ & $5.00990 E-03$ \\
\hline & & 1.0 & $1.0416 E+00$ & $4.16000 E-02$ & $1.0244 E+00$ & $2.44000 E-02$ & $1.0431 E+00$ & $4.31000 E-02$ \\
\hline
\end{tabular}

TABLE 4: The approximate values and the absolute relative error values using DKM method for nonlinear case $(k=2)$.

\begin{tabular}{|c|c|c|c|c|c|c|c|c|}
\hline \multirow{2}{*}{$N$} & \multirow{2}{*}{$t$} & \multirow{2}{*}{$x$} & \multicolumn{2}{|c|}{$v=0.21$} & \multicolumn{2}{|c|}{$v=0.27$} & \multicolumn{2}{|c|}{$v=0.33$} \\
\hline & & & Approx.DKM & Error $_{\text {DKM }}$ & Approx.DKM & Error $_{\mathrm{DKM}}$ & Approx.DKM & Error $_{\text {DKM }}$ \\
\hline \multirow{9}{*}{10} & \multirow{3}{*}{0.2} & 0.2 & $1.02397 E-07$ & $3.00000 E-12$ & $1.02396 E-07$ & $4.00000 E-12$ & $1.02395 E-07$ & $5.00000 E-12$ \\
\hline & & 0.6 & $2.23831 E-04$ & $1.17000 E-07$ & $2.23801 E-04$ & $1.47000 E-07$ & $2.23756 E-04$ & $1.92000 E-07$ \\
\hline & & 1.0 & $7.97493 E-03$ & $2.50700 E-05$ & $7.96913 E-03$ & $3.08700 E-05$ & $7.06090 E-03$ & $9.39100 E-04$ \\
\hline & \multirow{3}{*}{0.6} & 0.2 & $2.77078 E-06$ & $5.98000 E-09$ & $2.77274 E-06$ & $7.94000 E-09$ & $2.77611 E-06$ & $1.13100 E-08$ \\
\hline & & 0.6 & $6.05367 E-03$ & $7.06000 E-06$ & $6.05706 E-03$ & $1.04500 E-05$ & $6.06346 E-03$ & $1.68500 E-05$ \\
\hline & & 1.0 & $2.14910 E-01$ & $1.09000 E-03$ & $2.14854 E-01$ & $1.14600 E-03$ & $2.14879 E-01$ & $1.12100 E-03$ \\
\hline & \multirow{3}{*}{1} & 0.2 & $1.30309 E-05$ & $2.30900 E-07$ & $1.31058 E-05$ & $3.05800 \mathrm{E}-07$ & $1.32339 E-05$ & $4.33900 E-07$ \\
\hline & & 0.6 & $2.85827 E-02$ & $5.89100 E-04$ & $2.87835 E-02$ & $7.89900 E-04$ & $2.91304 E-02$ & $1.13680 E-03$ \\
\hline & & 1.0 & $1.0195 E+00$ & $1.95000 E-02$ & $1.0286 E+00$ & $2.86000 E-02$ & $1.0450 E+00$ & $4.50000 E-02$ \\
\hline \multirow{9}{*}{20} & \multirow{3}{*}{0.2} & 0.2 & $1.02397 E-07$ & $3.00000 E-12$ & $1.02396 E-07$ & $4.00000 E-12$ & $1.02395 E-07$ & $5.00000 E-12$ \\
\hline & & 0.6 & $2.23831 E-04$ & $1.17000 E-07$ & $2.23801 E-04$ & $1.47000 E-07$ & $2.23756 E-04$ & $1.92000 E-07$ \\
\hline & & 1.0 & $7.97493 E-03$ & $2.50700 E-05$ & $7.96913 E-03$ & $3.08700 E-05$ & $7.96090 E-03$ & $3.91000 E-05$ \\
\hline & \multirow{3}{*}{0.6} & 0.2 & $2.77078 E-06$ & $5.98000 E-09$ & $2.77274 E-06$ & $7.94000 E-09$ & $2.77611 E-06$ & $1.13100 E-08$ \\
\hline & & 0.6 & $6.05367 E-03$ & $7.06000 E-06$ & $6.05763 E-03$ & $1.10200 E-05$ & $6.06346 E-03$ & $1.68500 E-05$ \\
\hline & & 1.0 & $2.14910 E-01$ & $1.09000 E-03$ & $2.14854 E-01$ & $1.14600 E-03$ & $2.14879 E-01$ & $1.12100 E-03$ \\
\hline & \multirow{3}{*}{1} & 0.2 & $1.30309 E-05$ & $2.30900 E-07$ & $1.31058 E-05$ & $3.05800 E-07$ & $1.32339 E-05$ & $4.33900 E-07$ \\
\hline & & 0.6 & $2.85827 E-02$ & $5.89100 E-04$ & $2.87835 E-02$ & $7.89900 E-04$ & $2.91304 E-02$ & $1.13680 E-03$ \\
\hline & & 1.0 & $1.0952 E+00$ & $9.52000 E-02$ & $1.0286 E+00$ & $2.86000 E-02$ & $1.0450 E+00$ & $4.50000 E-02$ \\
\hline
\end{tabular}



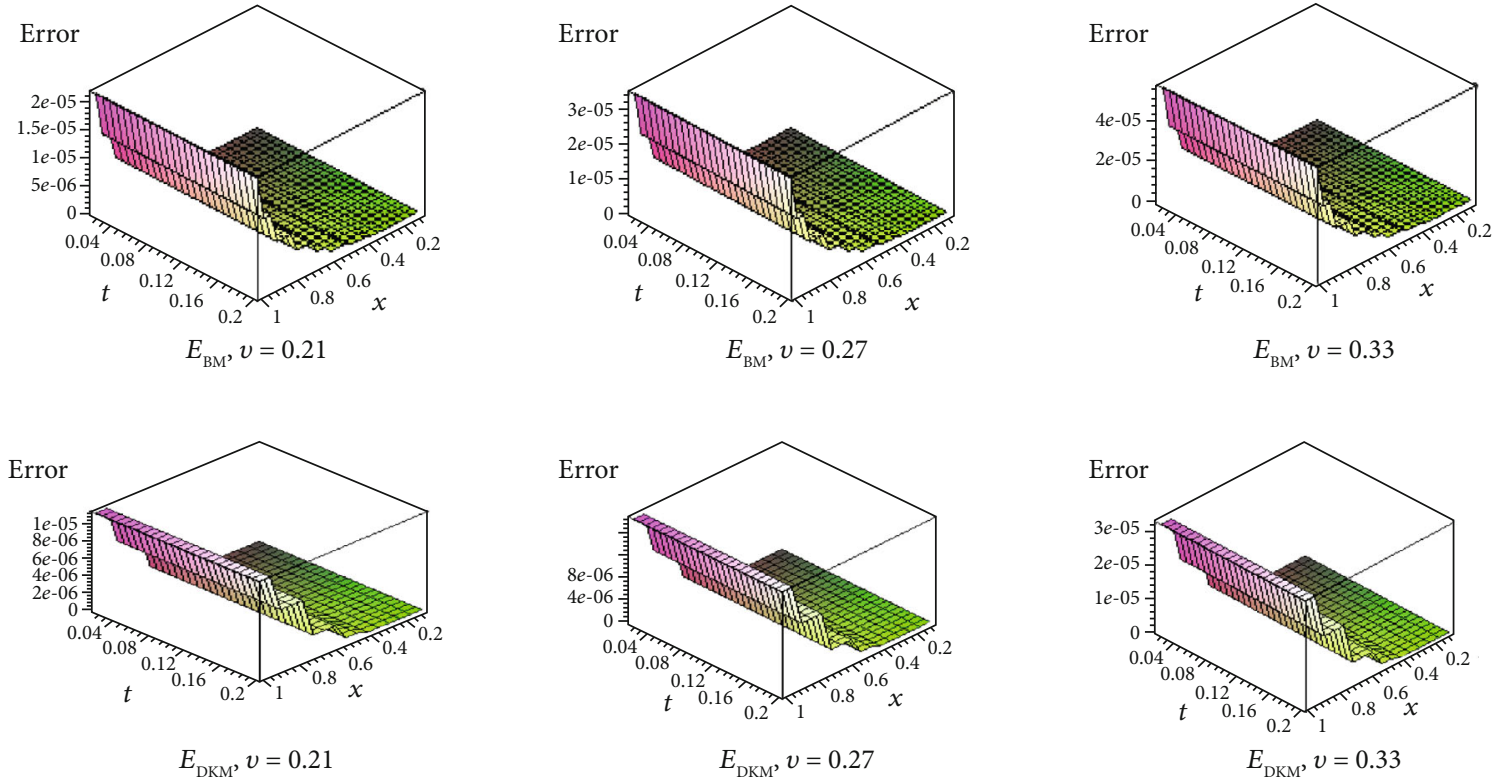

Figure 1: The values of errors by B-by-BM and DKM at $T=0.2, N=10$, and $k=1$ for $v=0.21,0.27,0.33$.
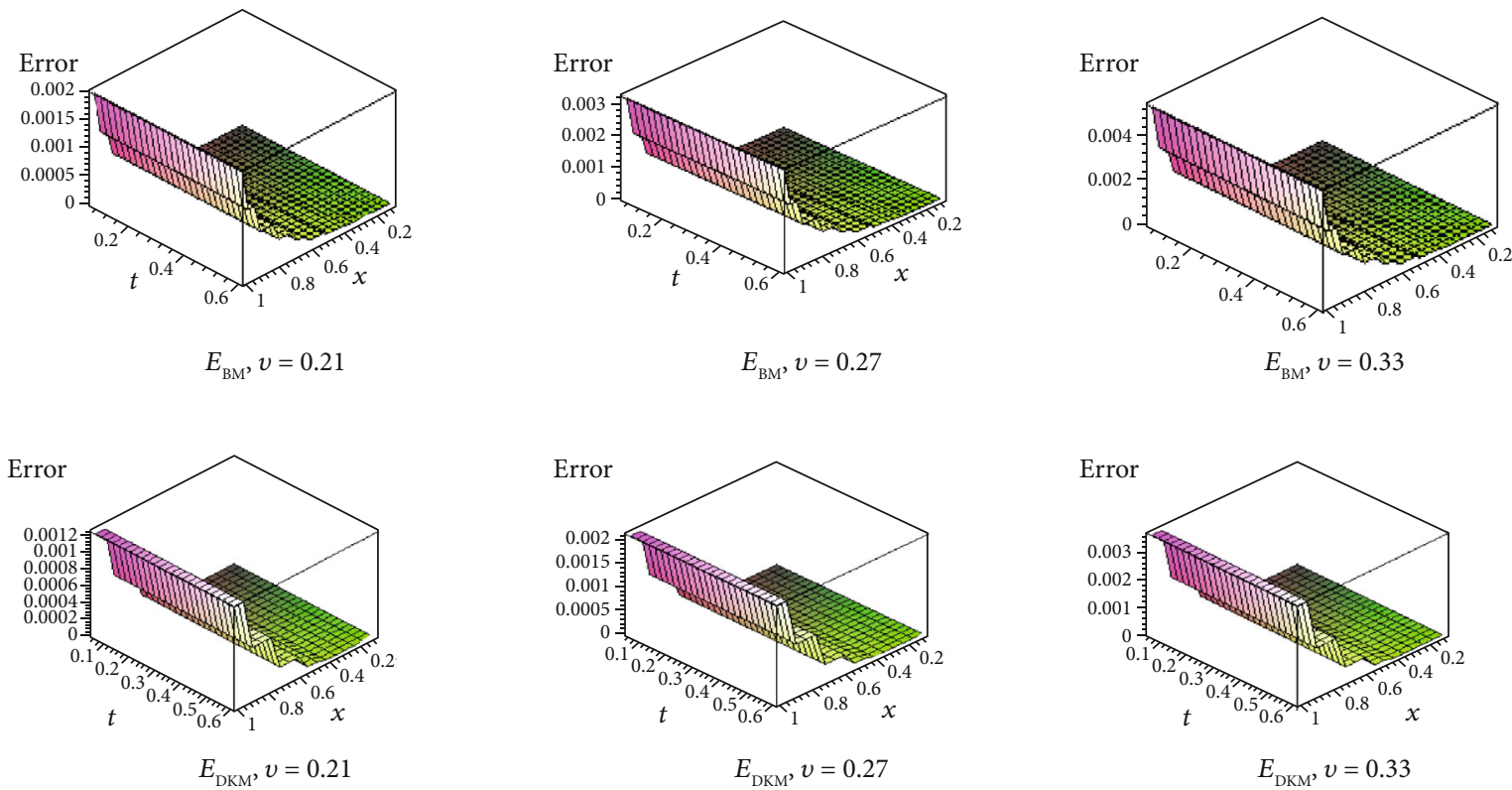

FIgure 2: The values of errors by B-by-BM and DKM at $T=0.6, N=10$, and $k=1$ for $v=0.21,0.27,0.33$.

\section{Solution of NT-DVIE}

We consider the bar in the nonlinear case; then, the integral equation (39) with continuous kernel reduced to

$\mu \alpha(x, t)=f(x, t)-\lambda \int_{0}^{t} \int_{0}^{x} k(|t-\tau|,|x-y|) \gamma(\tau, y, \alpha(y, \tau)) d y d \tau$,

where $\gamma(y, \tau, \alpha(y, \tau))$ and $f(x, t) \in L_{2}[0, b] \times C[0, T]$ are given continuous functions. $\lambda$, which have many physical meaning, may be complex. The kernel $k(|t-\tau|,|x-y|)$ is continuous.
5.1. The $B-b y-B M$. In this section, we use the B-by-BM for solving the NT-DVIE of the second kind.

The interval $[0, b]$ is divided into steps of width $h, x_{j}=j$ $h, j=0,1, \cdots, n$, and $h=(b-a) / n$. The approximate solution of $\alpha_{1}(x)$ will be defined at mesh points $x_{j}$ and denoted by $\alpha_{i j}, j=0,1, \cdots, n$, such as $\alpha_{i j}$ is an approximation to $\alpha_{i}\left(x_{j}\right)$.

To solve the NT-DVIE,

$$
U(t)=F(t)-\lambda \int_{0}^{t} \int_{0}^{x} G(t, \tau, x, y, U(y, \tau)) d y d \tau,
$$



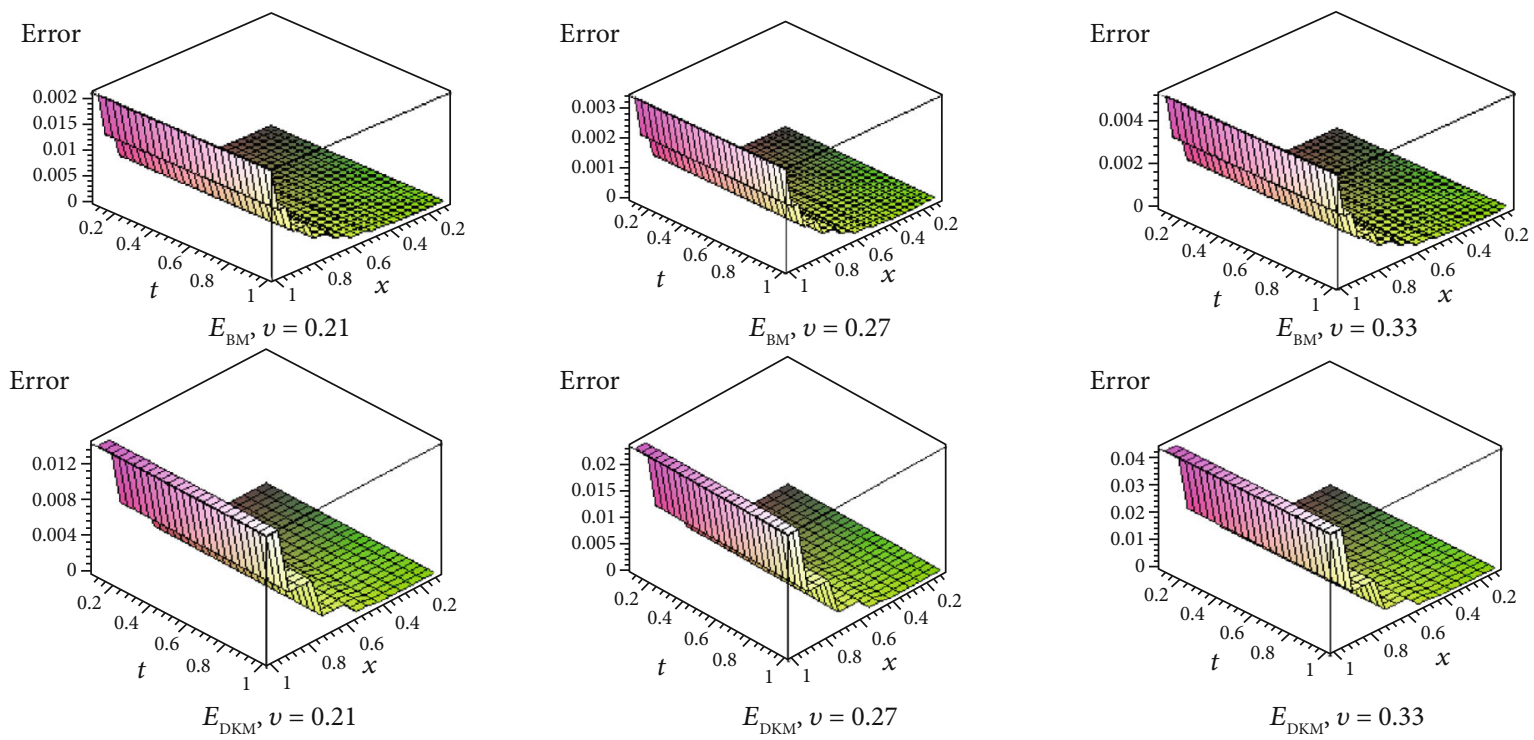

Figure 3: The values of errors by B-by-BM and DKM at $T=1, N=10$, and $k=1$ for $v=0.21,0.27,0.33$.

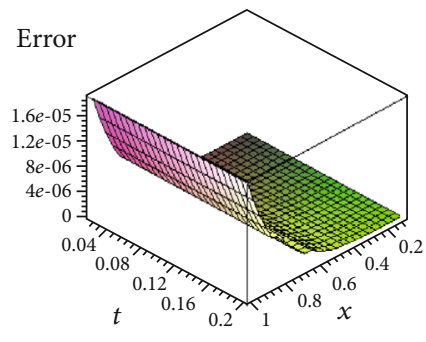

$E_{\mathrm{BM}}, v=0.21$

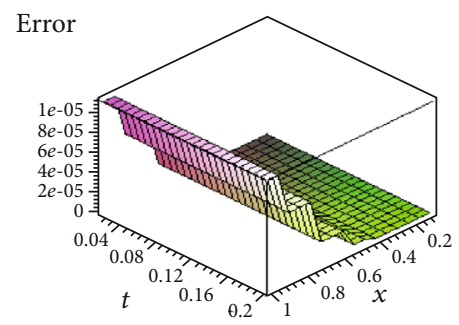

$E_{\mathrm{DKM}}, v=0.21$

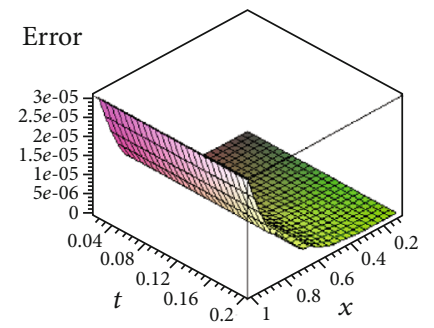

$E_{\mathrm{BM}}, v=0.27$

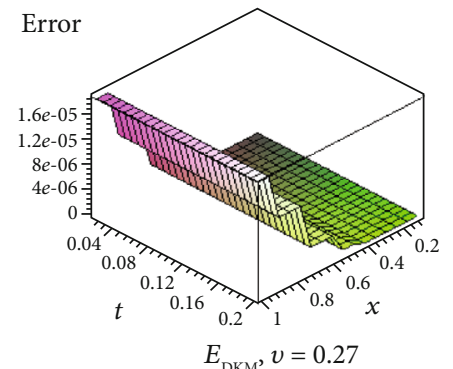

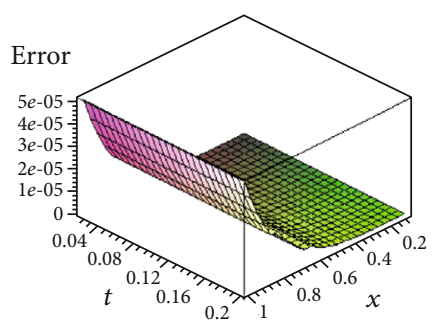

$E_{\mathrm{BM}}, v=0.33$

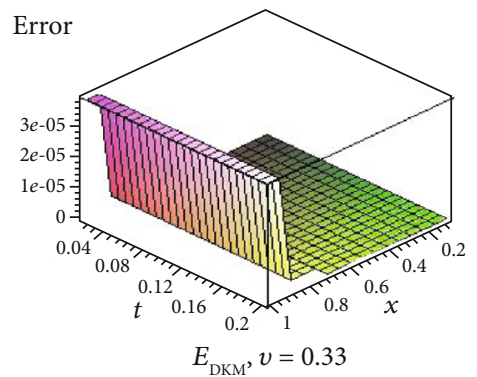

FIgure 4: The values of errors by B-by-BM and DKM at $T=0.2, N=20$, and $k=1$ for $v=0.21,0.27,0.33$. 

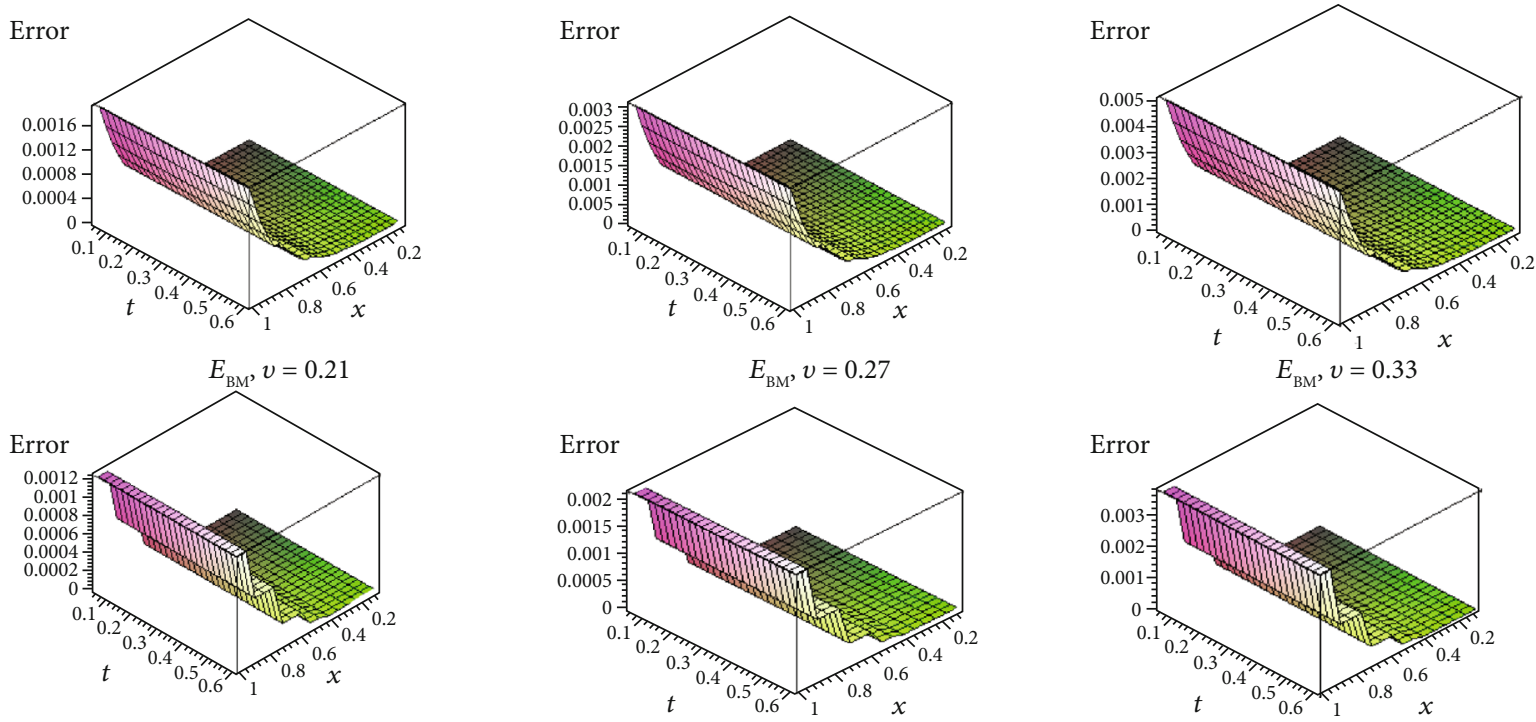

$$
E_{\mathrm{DKM}}, v=0.21
$$

$$
E_{\mathrm{DKM}}, v=0.27
$$

$E_{\mathrm{DKM}}, v=0.33$

Figure 5: The values of errors by B-by-BM and DKM at $T=0.6, N=20$, and $k=1$ for $v=0.21,0.27,0.33$.

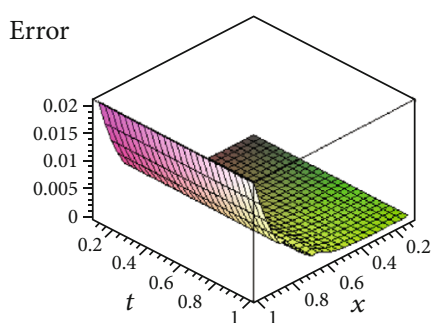

$$
E_{\mathrm{BM}}, v=0.21
$$

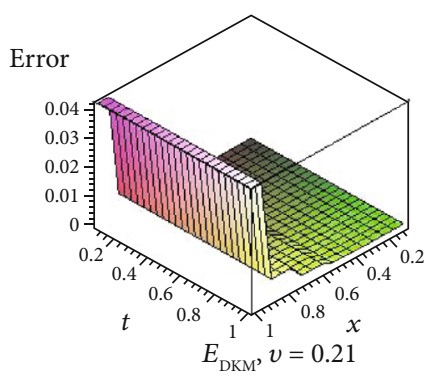

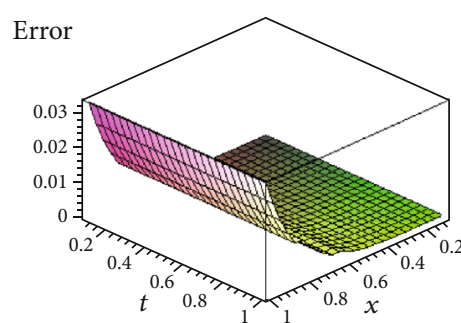

$E_{\mathrm{BM}}, v=0.27$

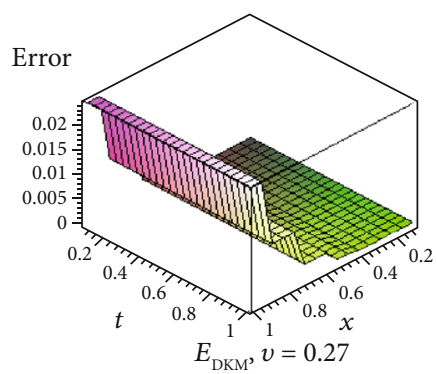

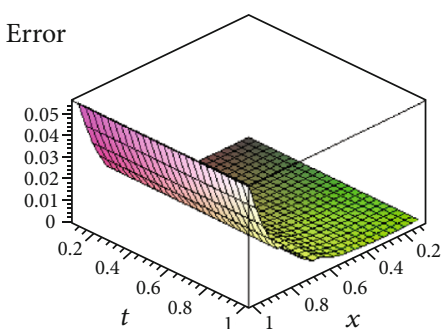

$E_{\mathrm{BM}}, v=0.33$

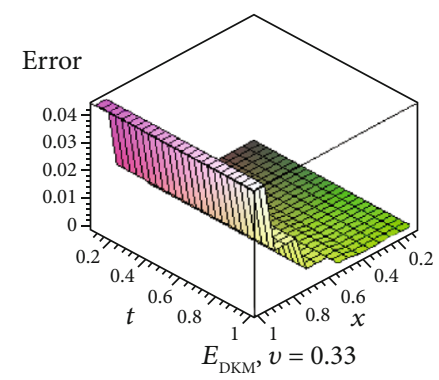

Figure 6: The values of errors by B-by- BM and DKM at $T=1, N=20$, and $k=1$ for $v=0.21,0.27,0.33$. 


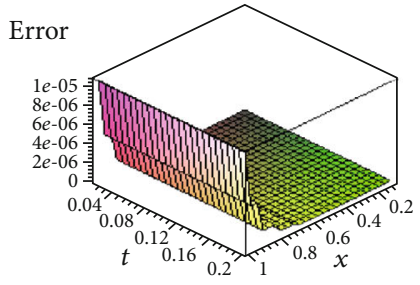

$E_{\mathrm{BM}}, v=0.21$

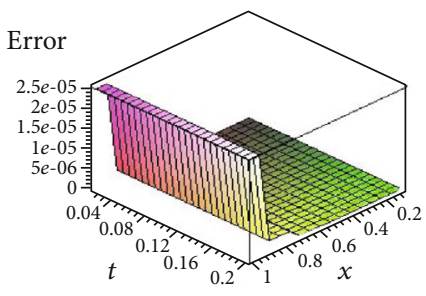

$E_{\mathrm{DKM}}, v=0.21$

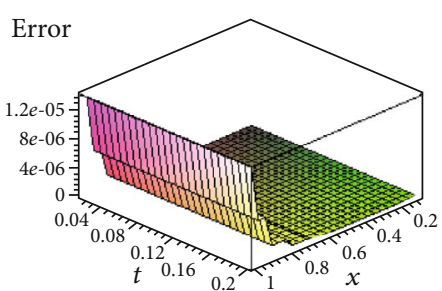

$E_{\mathrm{BM}}, v=0.27$

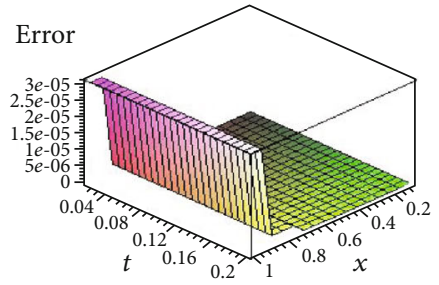

$E_{\mathrm{DKM}}, v=0.27$

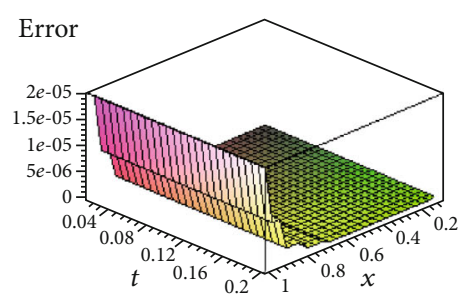

$E_{\mathrm{BM}}, v=0.33$

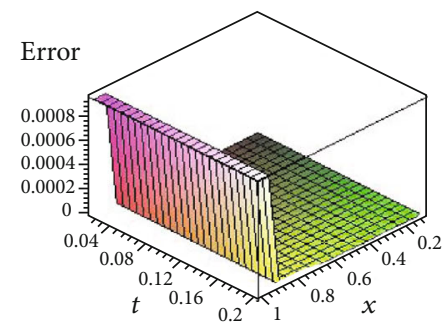

$E_{\mathrm{DKM}}, v=0.33$

Figure 7: The values of errors by B-by-BM and DKM at $T=0.2, N=10$, and $k=2$ for $v=0.21,0.27,0.33$.
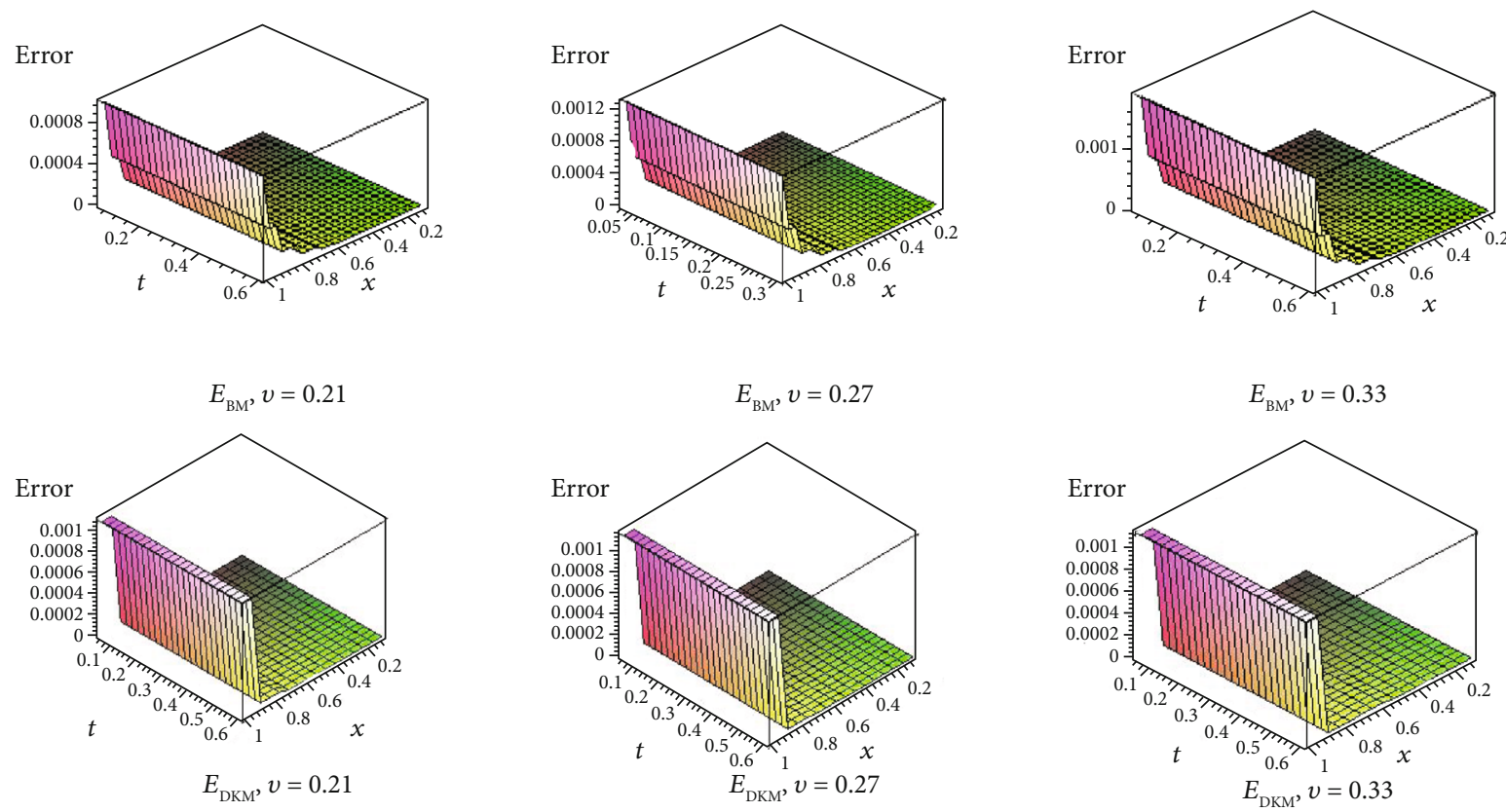

Figure 8: The values of errors by B-by-BM and DKM at $T=0.6, N=10$, and $k=2$ for $v=0.21,0.27,0.33$. 

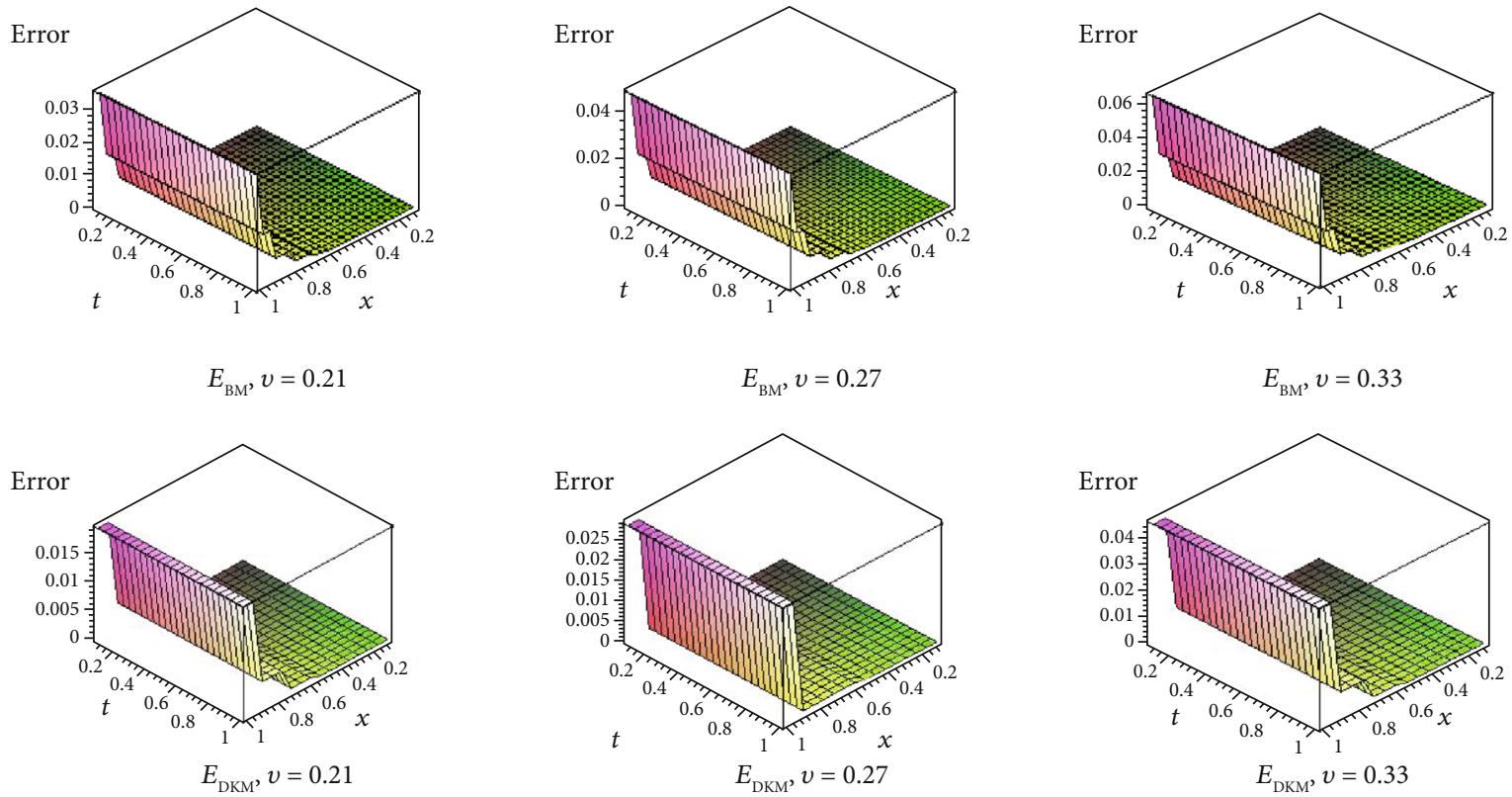

FIGURE 9: The values of errors by B-by-BM and DKM at $T=1, N=10$, and $k=2$ for $v=0.21,0.27,0.33$.

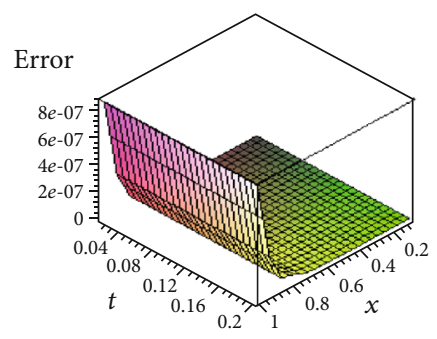

$E_{\mathrm{BM}}, v=0.21$

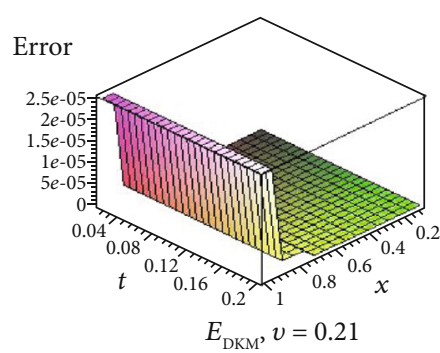

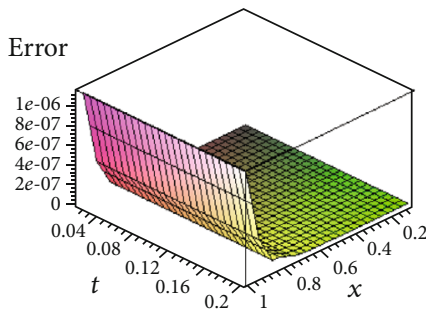

$E_{\mathrm{BM}}, v=0.27$

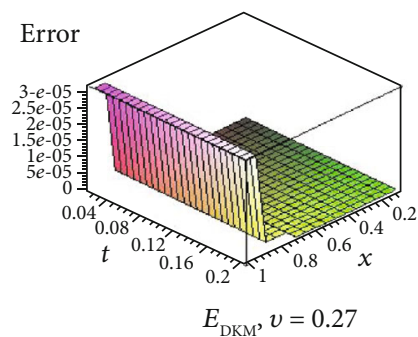

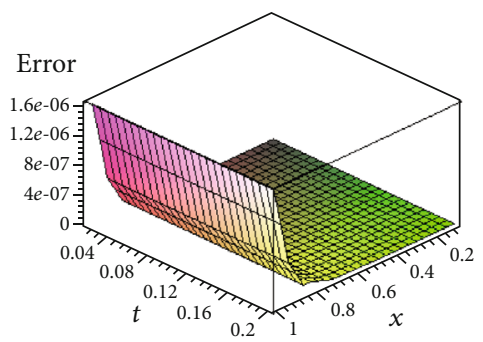

$E_{\mathrm{BM}}, v=0.33$

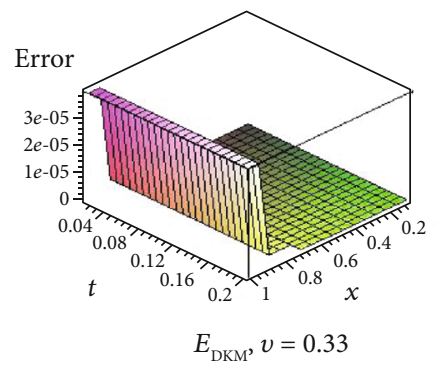

Figure 10: The values of errors by B-by-BM and DKM at $T=0.2, N=20$, and $k=2$ for $v=0.21,0.27,0.33$. 

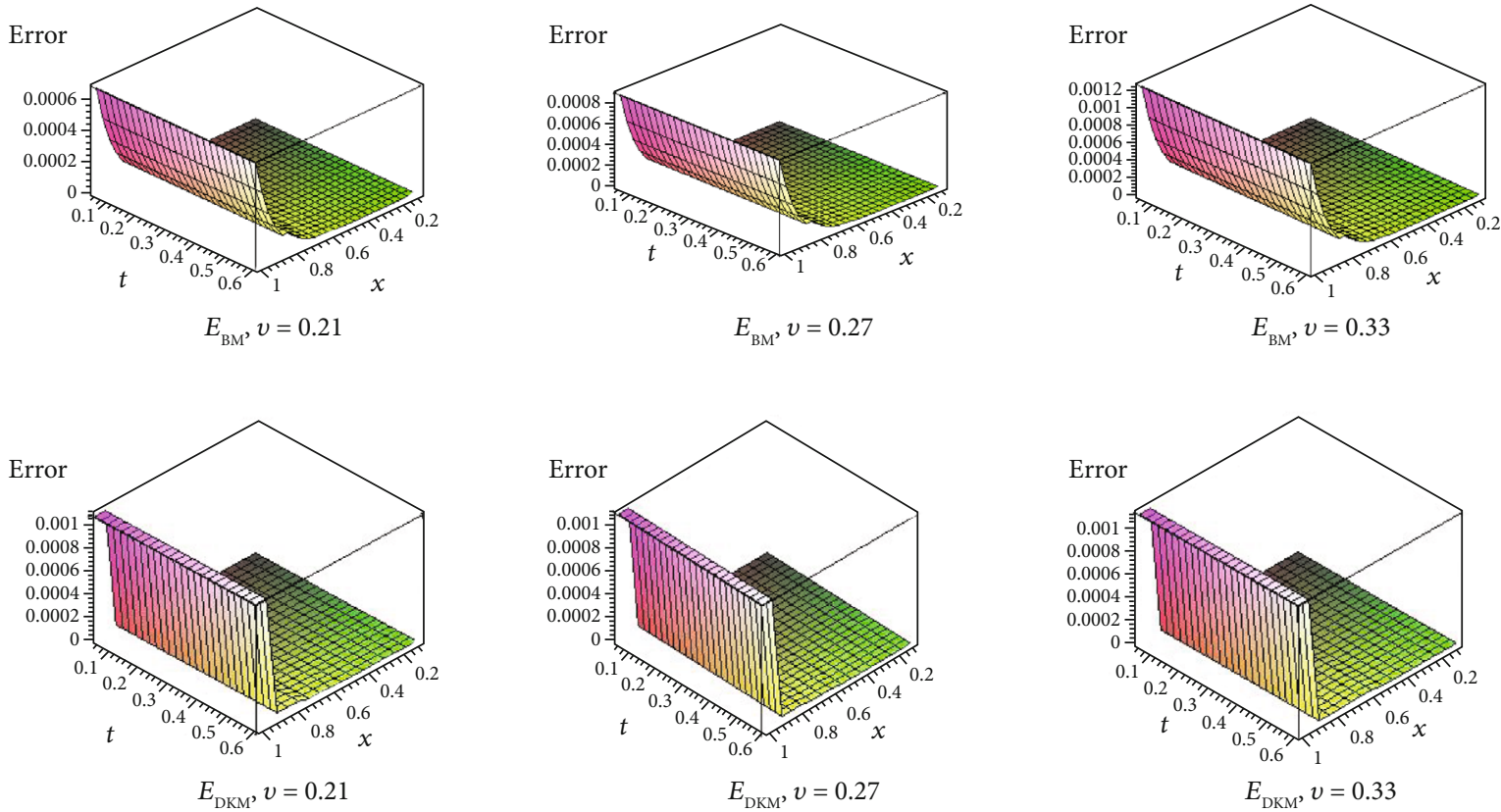

Figure 11: The values of errors by B-by-BM and DKM at $T=0.6, N=20$, and $k=2$ for $v=0.21,0.27,0.33$.
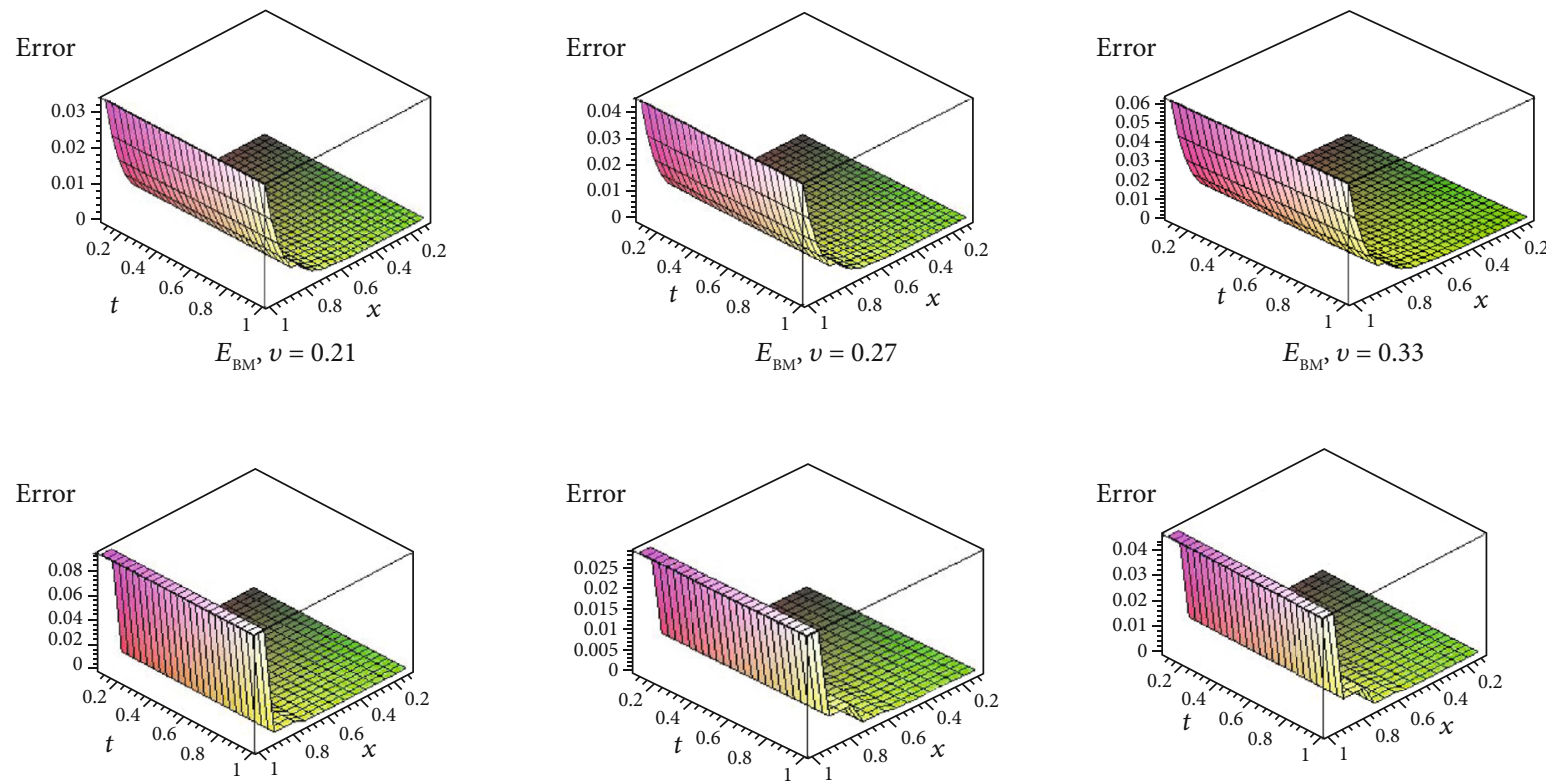

$$
E_{\mathrm{DKM}}, v=0.21
$$

$$
E_{\mathrm{DKM}}, v=0.27
$$$$
E_{\mathrm{DKM}}, v=0.33
$$

Figure 12: The values of errors by B-by-BM and DKM at $T=1, N=20$, and $k=2$ for $v=0.21,0.27,0.33$. 
where

$$
\begin{gathered}
U(t)=\left(\alpha_{1}(t), \cdots, \alpha_{l}(t)\right)^{T}, \\
U(t)=\left(\alpha_{1}(\tau), \cdots, \alpha_{l}(\tau)\right), \\
F(t)=\left(f_{1}(t), \cdots, f_{l}(t)\right)^{T}, \\
G(t, \tau, x, y, U(y, \tau))=G(t, \tau, x, y) \gamma(\tau, y, U(y, \tau)), \\
G(t, \tau, U(\tau))=\left[\begin{array}{ccc}
g_{1,1}(t, \tau, U(\tau)) & \cdots & g_{1, s}(t, \tau, U(\tau)) \\
\vdots & & \\
g_{s, 1}(t, \tau, U(\tau)) & \cdots & g_{s, s}(t, \tau, U(\tau))
\end{array}\right] .
\end{gathered}
$$

Then, we get

$$
\alpha_{n}+\lambda \int_{0}^{t} \int_{0}^{x} G(t, \tau, x, y, U(y, \tau)) d y d \tau
$$

Rewrite equation (58) as follows:

$$
\begin{aligned}
\alpha_{n}\left(t_{k}\right)= & f_{i}\left(t_{k}\right)-\lambda \int_{0}^{t_{p m}} \int_{0}^{x_{p m}} g_{i, s}\left(t_{k}, \tau, x_{k}, y, U\left(y_{1}, \tau\right)\right) d y d \tau \\
& -\lambda \int_{t_{p m}}^{t_{n}} \int_{x_{p m}}^{x_{n}} g_{i, s}\left(t_{k}, \tau, x_{k}, y, U\left(y_{1}, \tau\right)\right) d y d \tau .
\end{aligned}
$$

Here, $p$ is some integer and $m$ is $(|k / p|), s=1,2, \cdots$. If the values $\alpha_{i 0}, \alpha_{i 1}, \cdots \alpha_{i, n m}$, are known, then the first integral is obtained by a quadrature rule using values of the integrand at $\tau=t_{p m} ; t_{p m+1}, \cdots ; t_{p(m+1)} ; y=x_{p m} ; x_{p m+1}, \cdots ;$ and $x_{p(m+1)}$.

Then,

$$
\begin{aligned}
\alpha_{i k}= & f_{i}\left(x_{k}\right)-\lambda\left[h \sum_{j=0}^{m p} w_{k j} g_{i, s}\left(t_{k}, \tau_{j}, x_{k}, y_{j}, u_{1 j}, \cdots, u_{l j}\right)\right] \\
& -\lambda\left[h \sum_{j=m p}^{(m+1) p} w_{k j}^{*} g_{i, s}\left(t_{k}, \tau_{m p+j}, x_{k}, y_{m p+j}, u_{1, m p+j j}, \cdots, u_{l, m p+j}\right)\right],
\end{aligned}
$$

for $\quad n=m p+1, m p+2, \cdots,(m+1) p, m=0,1, \cdots,(N-1)$, where $w_{k j}, w^{*}{ }_{k j}$ depend on the quadrature rule used.

5.2. Modified Method of Two Blocks. For this method, we take $p=2$; the integration over $\left[a, t_{2 m}\right]$ can be accomplished by Simpson's rule, and the integral over $\left[t_{2 l}, t_{n}\right]$ can be accomplished by using a quadratic interpolation of the integrand at the point $t_{2 m}, t_{2 m+1}, t_{2 m+2}$; then, equation (58) becomes

$$
\alpha_{i, 2 m+1}=f_{i}\left(t_{2 m+1}\right)-\lambda \int_{a}^{(2 m+1) h} \int_{b}^{(2 m+1) h} g_{i, s}\left(t_{2 m+1}, \tau, x_{2 m+1}, y, U(y, \tau)\right) d y d \tau,
$$

$\alpha_{i, 2 m+2}=f_{i}\left(t_{2 m+2}\right)-\lambda \int_{a}^{(2 m+2) h} \int_{b}^{(2 m+2) h} g_{i, s}\left(t_{2 m+2}, \tau, x_{2 m+2}, y, U(y, \tau)\right) d y d \tau$,

where $i=1,2, \cdots, l, m=0,1, \cdots$.

On the other hand, from (59), equations (61) and (62) can be written as

$$
\begin{aligned}
\alpha_{i, 2 m+1}= & f_{i}\left(t_{2 m+1}\right)-\lambda \int_{a}^{2 m h} \int_{b}^{2 m h} g_{i, s}\left(t_{2 m+1}, \tau, x_{2 m+1}, y, U(y, \tau)\right) d y d \tau \\
& -\lambda \int_{2 m h}^{(2 m+1) h} \int_{2 m h}^{(2 m+1) h} g_{i, s}\left(t_{2 m+1}, \tau, x_{2 m+1}, y, U(y, \tau)\right) d y d \tau,
\end{aligned}
$$

$$
\begin{aligned}
\alpha_{i, 2 m+2}= & f_{i}\left(t_{2 m+2}\right)-\lambda \int_{a}^{2 m h} \int_{b}^{2 m h} g_{i, s}\left(t_{2 m+2}, \tau, x_{2 m+2}, y, U(y, \tau)\right) d y d \tau \\
& -\lambda \int_{2 m h}^{(2 m+1) h} \int_{2 m h}^{(2 m+1) h} g_{i, s}\left(t_{2 m+2}, \tau, x_{2 m+2}, y, U(y, \tau)\right) d y d \tau .
\end{aligned}
$$

Therefore, by equation (60), the approximate solution is computed by

$$
\begin{aligned}
\alpha_{i, 2 m+1}= & f_{i}\left(t_{2 m+1}\right)-\lambda\left[\frac{h}{3} \sum_{j=0}^{2 m} w_{j} g_{i, s}\left(t_{2 m+1}, \tau_{j}, x_{2 m+1}, y_{j}, u_{1 j}, \cdots, u_{l j}\right)\right. \\
& +\lambda\left\{\frac { h } { 1 2 } \left[5 g_{i, s}\left(t_{2 m+1}, \tau_{2 m}, x_{2 m+1}, y_{2 m}, u_{1,2 m}, \cdots, u_{l, 2 m}\right)\right.\right. \\
& +8 g_{i, s}\left(t_{2 m+1}, \tau_{2 m+1}, x_{2 m+1}, y_{2 m+1}, u_{1,2 m+1}, \cdots, u_{l, 2 m+1}\right) \\
& \left.\left.-g_{i, s}\left(t_{2 m+1}, \tau_{2 m+2}, x_{2 m+1}, y_{2 m+2}, u_{1,2 m+1}, \cdots, u_{l, 2 m+2}\right)\right]\right\},
\end{aligned}
$$

$\alpha_{i, 2 m+2}=f_{i}\left(t_{2 m+2}\right)-\lambda\left[\frac{h^{2 m+2}}{3} \sum_{j=0}^{2} w_{j}^{\prime} g_{i, s}\left(t_{2 m+2}, \tau_{j}, x_{2 m+2}, y_{j}, u_{1 j}, \cdots, u_{l j}\right)\right]$,

where $\alpha_{1,0}=f_{i}\left(t_{0}\right)$.

Thus, replace the second term in equation (64) by formulas (62) and (64), and then, we get

$$
\begin{aligned}
\alpha_{i, 2 m+1}= & f_{i}\left(t_{2 m+1}\right)-\lambda\left[\frac{h}{3} \sum_{j=0}^{2 m} w_{j} g_{i, s}\left(t_{2 m+1}, \tau_{j}, x_{2 m+1}, y_{j}, \alpha_{1 j}, \cdots, \alpha_{l j}\right)\right. \\
& ++\lambda\left\{\frac { h } { 6 } \left[g_{i, s}\left(t_{2 m+1}, \tau_{2 m}, x_{2 m+1}, y_{2 m}, \alpha_{1,2 m}, \cdots, \alpha_{l, 2 m}\right)\right.\right. \\
& +4 g_{i, s}\left(t_{2 m+1}, \tau_{2 m+1 / 2}, x_{2 m+1}, y_{2 m+1 / 2}\right. \\
& +\left(\frac{3}{8} \alpha_{1,2 m}+\frac{3}{4} \alpha_{i, 2 m+1}-\frac{1}{8} \alpha_{l, 2 m+2}\right) \\
& \left.\cdots,\left(\frac{3}{8} \alpha_{l, 2 m}+\frac{3}{4} \alpha_{l, 2 m+1^{-}} \frac{1}{8} \alpha_{l, 2 m+2}\right)\right) \\
& \left.\left.+g_{i, s}\left(t_{2 m+1}, \tau_{2 m+1}, x_{2 m+1}, y_{2 m+1}, \alpha_{1,2 m+1}, \cdots, \alpha_{l, 2 m+1}\right)\right]\right\}
\end{aligned}
$$




$$
\alpha_{i, 2 m+2}=f_{i}\left(t_{2 m+2}\right)-\lambda\left[\frac{h^{2 m+2}}{3} \sum_{j=0}^{\prime} w_{j}^{\prime} g_{i, s}\left(t_{2 m+2}, \tau_{j}, x_{2 m+2}, y_{j}, \alpha_{1 j}, \cdots, \alpha_{l j}\right)\right. \text {, }
$$

or

$$
\begin{aligned}
\alpha_{i, 2 m+2}= & f_{i}\left(t_{2 m+2}\right)-\lambda\left[\frac { h } { 3 } g _ { i , s } \left(t_{2 m+2}, \tau_{0}, x_{2 m+2}, y_{0},\left(\alpha_{10}, \cdots, \alpha_{l 0}\right)\right.\right. \\
& +4 g_{i, s}\left(t_{2 m+2}, \tau_{0}, x_{2 m+2}, y_{0},\left(\alpha_{11}, \cdots, \alpha_{l 1}\right)\right. \\
& \left.+\cdots+g_{i, s}\left(t_{2 m+2}, \tau_{2 m+2}, x_{2 m+2}, y_{2 m+2},\left(\alpha_{1,2 m+2}, \cdots, \alpha_{l, 2 m+2}\right)\right)\right],
\end{aligned}
$$

where

$$
\begin{gathered}
w_{0}=w_{2 m}=1, \\
w_{j}=3-(-1)^{j}, \quad j=1,2, \cdots, 2 m-1, \\
w_{0}^{\prime}=w_{2 m+2}^{\prime}=1, \\
w_{j}^{\prime}=3-(-1)^{j}, \quad j=1,2, \cdots, 2 m+1 .
\end{gathered}
$$

Finally, we construct $2 l$ linear equations from (67) and (68) to find the unknown functions $u_{i, 2 m+1}, u_{i, 2 m+2}$.

5.3. The DKM. In this part, we replace the given kernel $g(\mid t$ $-\tau|| x-y \mid$,$) approximately by a degenerate ker-$ nelg $g_{i}(|t-\tau|,|x-y|)$, that is,

$$
g_{i}(|t-\tau|,|x-y|)=\sum_{j=1}^{n} B_{j}^{\prime}(x) C_{j}(y) \sum_{i=1}^{n} B_{i}(t) C_{i}(\tau)
$$

such that

$$
\left\{\int_{0}^{t} \int_{0}^{t}\left|g(|t-\tau|,|x-y|)-g_{i}(|t-\tau|,|x-y|)\right|^{2} d t d \tau\right\}^{1 / 2} \longrightarrow 0 \quad \text { as } l \longrightarrow \infty
$$

Hence, the solution of equation (55) associated with the kernel $g_{i}(|t-\tau|,|x-y|)$ takes the form

$$
\mu \alpha(x, t)+\lambda \int_{0}^{t} \int_{0}^{x} g(|t-\tau|,|x-y|) \gamma(\tau, y, \alpha(y, \tau)) d y d \tau=f(x, t) .
$$

Using (70) in (72), we have

$$
\mu \alpha(x, t)+\lambda \sum_{i=0}^{n} \sum_{j=0}^{n} A_{i, j} B_{i}(t) B_{j}^{\prime}(x)=f(x, t),
$$

where

$$
A_{i, j}=\int_{0}^{t} \int_{0}^{x} C_{i}(\tau) C_{j}(y) \gamma(\tau, y, \beta(y, \tau)) d y d \tau
$$

Here, $A_{i, j}$ are constants to be determined from the follow- ing formula:

$$
A_{m, m}{ }^{\prime}=\int_{0}^{t} \int_{0}^{x} C_{m}(\tau) C_{m}{ }^{\prime}(y) \gamma\left(\tau, y, \frac{1}{\mu} f(y, \tau)-\frac{\lambda}{\mu} \sum_{i=1}^{n} \sum_{j=1}^{n} A_{i, j} B_{i}(\tau) B^{\prime}{ }_{j}(y)\right) d y d \tau,
$$

where $(j=0,1,2, \cdots n ; m=1,2, \cdots l)$. If we define

$$
\begin{aligned}
H_{m, m}\left(A_{1 m}{ }^{\prime}, A_{2 m}{ }^{\prime}, \cdots, A_{l m}{ }^{\prime}\right)= & \int_{0}^{t} \int_{0}^{x} C_{m}(\tau) C_{m}{ }^{\prime}(y) \gamma \\
& \cdot\left(\tau, y, \frac{1}{\mu} f(y, \tau)-\frac{\lambda}{\mu} \sum_{i=1}^{n} \sum_{j=1}^{n} A_{i, j} B_{i}(\tau) B^{\prime}{ }_{j}(y)\right) d y d \tau,
\end{aligned}
$$

$m=1,2, \cdots l$, then equation (75) can be solved numerically.

\section{Numerical Applications}

Consider

$\alpha(x, t)=f(x, t)-\lambda \int_{0}^{t} \int_{0}^{x} k(|t-\tau|,|x-y|)(\alpha(y, \tau))^{k} d y d \tau,(\mu=1)$.

We consider equation (77) in the linear and nonlinear case, where if $k=1$, we obtain the LTVIE, so in this case, we take $\lambda=\lambda^{*} / 2 G$ and $\lambda^{*}=2 G v /(1-2 v)$ also when $k \geq 2$. We obtain the NTVIE, and in this case, we take $\lambda=A_{2} K / 4$ $G A_{1}$ and $K=2 G(1+v) / 3(1-2 v)$, and we find $A_{1}$ and $A_{2}$ from equations (32) and (34); $a=4$ and $b=2$ are the major and the minor axes of the ellipse, respectively. In addition, we study three materials: plutonium $\lambda$ steel $v=0.27$, and Copper $v=0.33$, where $v$ is the Poisson ratio $0 \leq v \leq 1 / 2$.

Consider

$$
\alpha(x, t)=f(x, t)-\lambda \int_{0}^{t} \int_{0}^{x}(1+t \tau) y^{3}(\alpha(y, \tau))^{k} d y d \tau,(\mu=1),
$$

where the exact solution $\alpha(x, t)=x^{4} t^{3}$; B-by-BM and DKM are used to obtain the approximate numerical solutions and corresponding errors for materials: plutonium, steel, and copper, respectively, $N=10$ and 20 and time $t=0.2$ and $0.6,1$, respectively. Table 1 shows the approximate and the absolute relative error values for linear case. Table 2 shows the approximate and the absolute relative error values for nonlinear case. The codes were written in Maple 10 program.

In Tables 1 and 2, Approx. ${ }_{\text {BM }} \rightarrow$ approximate solution of B-by-BM and Error ${ }_{\mathrm{BM}} \rightarrow$ the absolute error of B-by-BM. In Tables 3 and 4, Approx.DKM $\rightarrow$ approximate solution of $\mathrm{DKM}$ and Error $_{\mathrm{DKM}} \rightarrow$ the absolute error of DKM.

\section{The Conclusions}

This paper deals with a new computational method for approximate solution of NT-DVIE of the second kind with continues kernels. For this purpose, B-by-BM and DKM 
has been presented to solution the problem. These methods have proven to be effective in solving an equation NTDVIE. Error analysis and some numerical examples are presented for different materials to illustrate the effectiveness and accuracy of the methods.

From the previous results in Tables 1 and 2 and Figures 1-12, we notice the following:

(1) When the values of $v$ and $\lambda$ are fixed in the linear and nonlinear case, then the error value increases with the time $x, t=0.2,0.6,1$

(2) In the linear and nonlinear case, when the values of time are fixed, the error value increases with the increase of $v$ and $\lambda$

(3) When the values of $v, \lambda$, and time $t$ are fixed, the error value decreases with $N$ which is increasing, for the linear and nonlinear case and for each material (plutonium, steel, and copper)

(4) As $x$ is increasing and $t$ is fixed, the errors are also increasing for the linear and nonlinear case and for each material

(5) The approximate solutions calculated by B-by-BM and DKM are best methods for LT-DVIE and NTDVIE

(6) In general, the maximum value of the errors by B-by$\mathrm{BM}$ and DKM in the linear case is less than the maximum value of the errors in the nonlinear case, for all materials, and the minimum value of the errors in the linear case is larger than the minimum value of the errors in the nonlinear case

(7) The previous numerical experiments illustrate the accuracy of the proposed methods to solve the problem

\section{Data Availability}

All the data are available within the article and also as the references that were cited.

\section{Conflicts of Interest}

The author declares that there are no conflicts of interest.

\section{References}

[1] N. I. Muskhelishvili, Some Basic Problems of Mathematical Theory of Elasticity, Noordhoff, Holland, 1953.

[2] P. Frank and R. Mises, Torsion in Mechanics and Physics, Dover, New York, 1961.

[3] J. L. Nowinski, Theory of Thermo Elasticity with Applications, Sijthoff \&Noordhoff international publishers, Alphen and Den Rijn, 1978.

[4] I. N. Sneddon and B. S. Berry, The Classical Theory of Elasticity, Springer, Berlin, 1968.

[5] E. J. Sapountzakis, "Solution of non-uniform torsion of bars by an integral equation method," Elsevier Science Ltd, 2000.
[6] E. J. Sapountzakis and V. J. Tsipiras, "Nonlinear inelastic uniform torsion of bars by BEM," Computational Mechanics, vol. 42, no. 1, pp. 77-94, 2008.

[7] E. J. Sapountzakis and V. J. Tsipiras, A BEM Solution to the Nonlinear Inelastic Uniform Torsion Problem of Composite Bars, Springer Netherlands, 2009.

[8] I. L. El-Kalla and A. M. Al-Bugami, "Numerical solution of nonlinear Volterra-Fredholm integral equation with applications in torsion problem," International Journal of Computational and Applied Mathematics, vol. 7, pp. 403-418, 2012.

[9] S. M. Sheshtawy and A. F. Ghaleb, "Approximate solution to the problem of torsion of an in nite elastic rod of normal cross-section in the form of a nephroid by a boundary integral method," Al Azhar Buletin of Science, vol. 25, no. 1, 2014.

[10] P. Assari, "On the numerical solution of two-dimensional integral equations using a meshless local discrete Galerkin scheme with error analysis," Engineering with Computers volume, vol. 35, no. 3, pp. 893-916, 2019.

[11] F. Fattahzadeh, "Approximate solution of two-dimensional Fredholm integral equation of the first kind using wavelet base method," International Journal of Applied and Computational Mathematics, vol. 5, p. 138, 2019.

[12] S. M. Torabi and A. Tari, "Numerical solution of twodimensional IE of the first kind by multi-step method," Computational Methods for Differential Equations, vol. 4, no. 2, pp. 128-138, 2016.

[13] A. Alturk, "The regularization-homotopy method for the twodimensional Fredholm integral equations of the first kind," Mathematical and Computational Applications, vol. 21, p. 9, 2016. 